\title{
A note for some parabolic multilinear commutators generated by a class of parabolic maximal and linear operators with rough kernel on the parabolic generalized local Morrey spaces
}

\author{
Ferit Gürbüz(D) \\ Hakkari University, Faculty of Education, Department of Mathematics Education, Hakkari 30000, Turkey
}

\begin{abstract}
In this paper, we give the boundedness of some parabolic multilinear commutators generated by a class of parabolic maximal and linear operators with rough kernel and parabolic local Campanato functions on the parabolic generalized local Morrey spaces, respectively. Indeed, the results in this paper are extensions of some known results.
\end{abstract}

Mathematics Subject Classification (2010). 42B20, 42B25

Keywords. parabolic multilinear commutators, rough kernel, parabolic generalized local Morrey space, parabolic local Campanato space

\section{Introduction and main results}

Let $S^{n-1}=\left\{x \in \mathbb{R}^{n}:|x|=1\right\}$ denote the unit sphere on $\mathbb{R}^{n}(n \geq 2)$ equipped with the normalized Lebesgue measure $d \sigma\left(x^{\prime}\right)$, where $x^{\prime}$ denotes the unit vector in the direction of $x$ and $\alpha_{n} \geq \alpha_{n-1} \geq \cdots \geq \alpha_{1} \geq 1$ be fixed real numbers.

Note that for each fixed $x=\left(x_{1}, \ldots, x_{n}\right) \in \mathbb{R}^{n}$, the function

$$
F(x, \rho)=\sum_{i=1}^{n} \frac{x_{i}^{2}}{\rho^{2 \alpha_{i}}}
$$

is a strictly decreasing function of $\rho>0$. Hence, there exists a unique $\rho=\rho(x)$ such that $F(x, \rho)=1$. It is clear that for each fixed $x \in \mathbb{R}^{n}$, the function $F(x, \rho)$ is a decreasing function in $\rho>0$. Fabes and Riviére [5] showed that $\left(\mathbb{R}^{n}, \rho\right)$ is a metric space which is often called the mixed homogeneity space related to $\left\{\alpha_{i}\right\}_{i=1}^{n}$. For $t>0$, we let $A_{t}$ be the diagonal $n \times n$ matrix

$$
A_{t}=\operatorname{diag}\left[t^{\alpha_{1}}, \ldots, t^{\alpha_{n}}\right]=\left(\begin{array}{ccc}
t^{\alpha_{1}} & & 0 \\
& \ddots & \\
0 & & t^{\alpha_{n}}
\end{array}\right) .
$$

Email address: feritgurbuz@hakkari.edu.tr Received: 26.07.2017; Accepted: 06.02.2019 
Let $\rho \in(0, \infty)$ and $0 \leq \varphi_{n-1} \leq 2 \pi, 0 \leq \varphi_{i} \leq \pi, i=1, \ldots, n-2$. For any $x=$ $\left(x_{1}, x_{2}, \ldots, x_{n}\right) \in \mathbb{R}^{n}$, set

$$
\begin{aligned}
x_{1} & =\rho^{\alpha_{1}} \cos \varphi_{1} \ldots \cos \varphi_{n-2} \cos \varphi_{n-1}, \\
x_{2} & =\rho^{\alpha_{2}} \cos \varphi_{1} \ldots \cos \varphi_{n-2} \sin \varphi_{n-1}, \\
& \vdots \\
x_{n-1} & =\rho^{\alpha_{n-1}} \cos \varphi_{1} \sin \varphi_{2}, \\
x_{n} & =\rho^{\alpha_{n}} \sin \varphi_{1} .
\end{aligned}
$$

Thus $d x=\rho^{\alpha-1} J\left(x^{\prime}\right) d \rho d \sigma\left(x^{\prime}\right)$, where $\alpha=\sum_{i=1}^{n} \alpha_{i}, x^{\prime} \in S^{n-1}, J\left(x^{\prime}\right)=\sum_{i=1}^{n} \alpha_{i}\left(x_{i}^{\prime}\right)^{2}, d \sigma$ is the element of area of $S^{n-1}$ and $\rho^{\alpha-1} J\left(x^{\prime}\right)$ is the Jacobian of the above transform. It is easy to see that $J\left(x^{\prime}\right) \in C^{\infty}\left(S^{n-1}\right)$ with $1 \leq J\left(x^{\prime}\right) \leq M$ and $x^{\prime} \in S^{n-1}$ for some $M \geq 1$.

Let $P$ be a real $n \times n$ matrix, whose all the eigenvalues have positive real part. Let $A_{t}=t^{P}(t>0)$, and set $\gamma=\operatorname{tr} P$. Then, there exists a quasi-distance $\rho$ associated with $P$ such that (see [3])

$(1-1) \rho\left(A_{t} x\right)=t \rho(x), t>0$, for every $x \in \mathbb{R}^{n}$,

$(1-2) \rho(0)=0, \rho(x-y)=\rho(y-x) \geq 0$, and $\rho(x-y) \leq k(\rho(x-z)+\rho(y-z))$,

$(1-3) d x=\rho^{\gamma-1} d \sigma(w) d \rho$, where $\rho=\rho(x), w=A_{\rho^{-1}} x$ and $d \sigma(w)$ is a measure on the unit ellipsoid $\{w: \rho(w)=1\}$.

Then, $\left\{\mathbb{R}^{n}, \rho, d x\right\}$ becomes a space of homogeneous type in the sense of Coifman-Weiss (see [3]) and a homogeneous group in the sense of Folland-Stein (see [6]).

Denote by $E(x, r)$ the ellipsoid with center at $x$ and radius $r$, more precisely, $E(x, r)=$ $\left\{y \in \mathbb{R}^{n}: \rho(x-y)<r\right\}$. Moreover, by the property of $\rho$ and the polar coordinates transform above, we have

$$
|E(x, r)|=\int_{\rho(x-y)<r} d y=v_{\rho} r^{\alpha_{1}+\cdots+\alpha_{n}}=v_{\rho} r^{\gamma},
$$

where $|E(x, r)|$ stands for the Lebesgue measure of $E(x, r)$ and $v_{\rho}$ is the volume of the unit ellipsoid on $\mathbb{R}^{n}$. By $E^{C}(x, r)=\mathbb{R}^{n} \backslash E(x, r)$, we denote the complement of $E(x, r)$. If we take $\alpha_{1}=\cdots=\alpha_{n}=1$ and $P=I$, then obviously $\rho(x)=|x|=\left(\sum_{i=1}^{n} x_{i}^{2}\right)^{\frac{1}{2}}, \gamma=n$, $\left(\mathbb{R}^{n}, \rho\right)=\left(\mathbb{R}^{n},|\cdot|\right), E_{I}(x, r)=B(x, r), A_{t}=t I$ and $J\left(x^{\prime}\right) \equiv 1$. Moreover, in the standard parabolic case $P_{0}=\operatorname{diag}[1, \ldots, 1,2]$ we have

$$
\rho(x)=\sqrt{\frac{\left|x^{\prime}\right|^{2}+\sqrt{\left|x^{\prime}\right|^{4}+x_{n}^{2}}}{2}}, \quad x=\left(x^{\prime}, x_{n}\right) .
$$

Note that we deal not exactly with the parabolic metric, but with a general anisotropic metric $\rho$ of generalized homogeneity, the parabolic metric being its particular case, but we keep the term parabolic in the title and text of the paper, the above existing tradition, see for instance [2].

Suppose that $\Omega(x)$ is a real-valued and measurable function defined on $\mathbb{R}^{n}$. Suppose that $S^{n-1}$ is the unit sphere on $\mathbb{R}^{n}(n \geq 2)$ equipped with the normalized Lebesgue surface measure $d \sigma$. Let $\Omega \in L_{s}\left(S^{n-1}\right)$ with $1<s \leq \infty$ be homogeneous of degree zero with respect to $A_{t}\left(\Omega(x)\right.$ is $A_{t}$-homogeneous of degree zero), that is, $\Omega\left(A_{t} x\right)=\Omega(x)$, for any $t>0, x \in \mathbb{R}^{n}$. We define $s^{\prime}=\frac{s}{s-1}$ for any $s>1$. One of the important problems on parabolic homogeneous spaces investigates the boundedness of parabolic linear operators satisfying the following size conditions $((1.1)$ and (1.2)). Therefore, in this paper, we consider parabolic linear operators $T_{\Omega}^{P}$ and $T_{\Omega, \alpha}^{P}, \alpha \in(0, \gamma)$ satisfying the size conditions 
for any $f \in L_{1}\left(\mathbb{R}^{n}\right)$ with compact support and $x \notin$ suppf , respectively

$$
\begin{gathered}
\left|T_{\Omega}^{P} f(x)\right| \leq c_{0} \int_{\mathbb{R}^{n}} \frac{|\Omega(x-y)|}{\rho(x-y)^{\gamma}}|f(y)| d y, \\
\left|T_{\Omega, \alpha}^{P} f(x)\right| \leq c_{0} \int_{\mathbb{R}^{n}} \frac{|\Omega(x-y)|}{\rho(x-y)^{\gamma-\alpha}}|f(y)| d y,
\end{gathered}
$$

where $c_{0}$ is independent of $f$ and $x$.

We point out that the conditions (1.1) and (1.2) in the case $\Omega \equiv 1, \alpha=0$ and $P=I$ was first introduced by Soria and Weiss in [11]. Indeed, in 1944, Soria and Weiss developed Stein's result [12] in the above shape. The conditions (1.1) and (1.2) are satisfied by many interesting operators in harmonic analysis, such as the parabolic Calderón-Zygmund operators, parabolic Carleson's maximal operator, parabolic Hardy-Littlewood maximal operator, parabolic C. Fefferman's singular multipliers, parabolic R. Fefferman's singular integrals, parabolic Ricci-Stein's oscillatory singular integrals, parabolic the BochnerRiesz means, the parabolic fractional integral operator(parabolic Riesz potential), parabolic fractional maximal operator, parabolic fractional Marcinkiewicz operator and so on (see $[7,8,11]$ for details).

The parabolic fractional maximal function $M_{\Omega, \alpha}^{P} f$ and $T_{\Omega, \alpha}^{p} f$ by with rough kernels, $0<\alpha<\gamma$, of a function $f \in L^{l o c}\left(\mathbb{R}^{n}\right)$ are defined by

$$
\begin{gathered}
M_{\Omega, \alpha}^{P} f(x)=\sup _{t>0}|E(x, t)|^{-1+\frac{\alpha}{\gamma}} \int_{E(x, t)}|\Omega(x-y)||f(y)| d y, \\
T_{\Omega, \alpha}^{P} f(x)=\int_{\mathbb{R}^{n}} \frac{\Omega(x-y)}{\rho(x-y)^{\gamma-\alpha}} f(y) d y,
\end{gathered}
$$

satisfy condition (1.2). It is obvious that when $\Omega \equiv 1, M_{1, \alpha}^{P} \equiv M_{\alpha}^{p}$ and $T_{1, \alpha}^{P} \equiv T_{\alpha}^{P}$ are the parabolic fractional maximal operator and the parabolic fractional integral operator, respectively. If $P=I$, then $M_{\Omega, \alpha}^{I} \equiv M_{\Omega, \alpha}$ and $T_{\Omega, \alpha}^{I} \equiv T_{\Omega, \alpha}$ are the fractional maximal operator with rough kernel and fractional integral operator with rough kernel, respectively. It is well known that the parabolic fractional maximal and integral operators play an important role in harmonic analysis (see $[2,6,8]$ ).

We notice that when $\alpha=0$, the above operators become the parabolic CalderónZygmund singular integral operator with rough kernel $T_{\Omega}^{P}=T_{\Omega, 0}^{P}$ and the corresponding parabolic maximal operator with rough kernel $M_{\Omega, 0}^{P} \equiv M_{\Omega}^{P}$ :

$$
\begin{gathered}
T_{\Omega}^{P} f(x)=p \cdot v \cdot \int_{\mathbb{R}^{n}} \frac{\Omega(x-y)}{\rho(x-y)^{\gamma}} f(y) d y, \\
M_{\Omega}^{P} f(x)=\sup _{t>0}|E(x, t)|^{-1} \int_{E(x, t)}|\Omega(x-y)||f(y)| d y,
\end{gathered}
$$

satisfy condition (1.1). It is obvious that when $\Omega \equiv 1, T_{\Omega}^{P} \equiv T^{P}$ and $M_{\Omega}^{P} \equiv M^{P}$ are the parabolic singular operator and the parabolic maximal operator, respectively. If $P=I$, then $M_{\Omega}^{I} \equiv M_{\Omega}$ is the Hardy-Littlewood maximal operator with rough kernel, and $T_{\Omega}^{I} \equiv T_{\Omega}$ is the homogeneous singular integral operator. It is well known that the parabolic maximal and singular operators play an important role in harmonic analysis (see $[2,6,7,14]$ ).

On the other hand let $b$ be a locally integrable function on $\mathbb{R}^{n}$, then for $0<\alpha<\gamma$, we define commutators generated by parabolic fractional maximal and integral operators 
with rough kernel and $b$ as follows, respectively.

$$
\begin{gathered}
M_{\Omega, b, \alpha}^{P}(f)(x)=\sup _{t>0}|E(x, t)|^{-1+\frac{\alpha}{\gamma}} \int_{E(x, t)}|b(x)-b(y)||\Omega(x-y)||f(y)| d y, \\
{\left[b, T_{\Omega, \alpha}^{P}\right] f(x) \equiv b(x) T_{\Omega, \alpha}^{P} f(x)-T_{\Omega, \alpha}^{P}(b f)(x)=\int_{\mathbb{R}^{n}}[b(x)-b(y)] \frac{\Omega(x-y)}{\rho(x-y)^{\gamma-\alpha}} f(y) d y .}
\end{gathered}
$$

Similarly, for $\alpha=0$, we define commutators generated by parabolic maximal and singular integral operators by with rough kernels and $b$ as follows, respectively.

$$
\begin{gathered}
M_{\Omega, b}^{P}(f)(x)=\sup _{t>0}|E(x, t)|^{-1} \int_{E(x, t)}|b(x)-b(y)||\Omega(x-y)||f(y)| d y, \\
{\left[b, T_{\Omega}^{P}\right] f(x) \equiv b(x) T_{\Omega}^{P} f(x)-T_{\Omega}^{P}(b f)(x)=p \cdot v \cdot \int_{\mathbb{R}^{n}}[b(x)-b(y)] \frac{\Omega(x-y)}{\rho(x-y)^{\gamma}} f(y) d y .}
\end{gathered}
$$

Because of the need for the study of partial differential equations (PDEs), Morrey [10] introduced Morrey spaces $M_{p, \lambda}$ which naturally are generalizations of Lebesgue spaces. We also refer to [1] for the latest research on the theory of Morrey spaces associated with harmonic analysis.

A measurable function $f \in L_{p}\left(\mathbb{R}^{n}\right), p \in(1, \infty)$, belongs to the parabolic Morrey spaces $M_{p, \lambda, P}\left(\mathbb{R}^{n}\right)$ with $\lambda \in[0, \gamma)$ if the following norm is finite:

$$
\|f\|_{M_{p, \lambda, P}}=\left(\sup _{x \in \mathbb{R}^{n}, r>0} \frac{1}{r^{\lambda}} \int_{E(x, r)}|f(y)|^{p} d y\right)^{1 / p},
$$

where $E(x, r)$ stands for any ellipsoid with center at $x$ and radius $r$. When $\lambda=0$, $M_{p, \lambda, P}\left(\mathbb{R}^{n}\right)$ coincides with the parabolic Lebesgue space $L_{p, P}\left(\mathbb{R}^{n}\right)$.

If $P=I$, then $M_{p, \lambda, I}\left(\mathbb{R}^{n}\right) \equiv M_{p, \lambda}\left(\mathbb{R}^{n}\right)$ and $L_{p, I}\left(\mathbb{R}^{n}\right) \equiv L_{p}\left(\mathbb{R}^{n}\right)$ are the classical Morrey and the Lebesgue spaces, respectively.

We now recall the definition of parabolic generalized local (central) Morrey space $L M_{p, \varphi, P}^{\left\{x_{0}\right\}}$ in the following.

Definition 1.1 (parabolic generalized local (central) Morrey space, [7, 8]). Let $\varphi(x, r)$ be a positive measurable function on $\mathbb{R}^{n} \times(0, \infty)$ and $1 \leq p<\infty$. For any fixed $x_{0} \in \mathbb{R}^{n}$ we denote by $L M_{p, \varphi, P}^{\left\{x_{0}\right\}} \equiv L M_{p, \varphi, P}^{\left\{x_{0}\right\}}\left(\mathbb{R}^{n}\right)$ the parabolic generalized local Morrey space, the space of all functions $f \in L_{p}^{l o c}\left(\mathbb{R}^{n}\right)$ with finite quasinorm

$$
\|f\|_{L M_{p, \varphi, P}^{\left\{x_{0}\right\}}}=\sup _{r>0} \varphi\left(x_{0}, r\right)^{-1}\left|E\left(x_{0}, r\right)\right|^{-\frac{1}{p}}\|f\|_{L_{p}\left(E\left(x_{0}, r\right)\right)}<\infty .
$$

According to this definition, we recover the local parabolic Morrey space $L M_{p, \lambda, P}^{\left\{x_{0}\right\}}$ and weak local parabolic Morrey space $W L M_{p, \lambda, P}^{\left\{x_{0}\right\}}$ under the choice $\varphi\left(x_{0}, r\right)=r^{\frac{\lambda-\gamma}{p}}$ :

$$
L M_{p, \lambda, P}^{\left\{x_{0}\right\}}=\left.L M_{p, \varphi, P}^{\left\{x_{0}\right\}}\right|_{\varphi\left(x_{0}, r\right)=r} \frac{\lambda-\gamma}{p}, \quad W L M_{p, \lambda, P}^{\left\{x_{0}\right\}}=\left.W L M_{p, \varphi, P}^{\left\{x_{0}\right\}}\right|_{\varphi\left(x_{0}, r\right)=r} \frac{\lambda-\gamma}{p} .
$$

Now, let us recall the defination of the space of $L C_{p, \lambda, P}^{\left\{x_{0}\right\}}$ (parabolic local Campanato space). 
Definition $1.2([7,8])$. Let $1 \leq p<\infty$ and $0 \leq \lambda<\frac{1}{\gamma}$. A parabolic local Campanato function $b \in L_{p}^{\text {loc }}\left(\mathbb{R}^{n}\right)$ is said to belong to the $L C_{p, \lambda, P}^{\left\{x_{0}\right\}}\left(\mathbb{R}^{n}\right)$, if

$$
\|b\|_{L C_{p, \lambda, P}^{\left\{x_{0}\right\}}}=\sup _{r>0}\left(\frac{1}{\left|E\left(x_{0}, r\right)\right|^{1+\lambda p}} \int_{E\left(x_{0}, r\right)}\left|b(y)-b_{E\left(x_{0}, r\right)}\right|^{p} d y\right)^{\frac{1}{p}}<\infty,
$$

where

$$
b_{E\left(x_{0}, r\right)}=\frac{1}{\left|E\left(x_{0}, r\right)\right|} \int_{E\left(x_{0}, r\right)} b(y) d y
$$

Define

$$
L C_{p, \lambda, P}^{\left\{x_{0}\right\}}\left(\mathbb{R}^{n}\right)=\left\{b \in L_{p}^{l o c}\left(\mathbb{R}^{n}\right):\|b\|_{L C_{p, \lambda, P}^{\left\{x_{0}\right\}}}<\infty\right\} .
$$

Let $b_{i}(i=1, \ldots, m)$ be locally integrable functions on $\mathbb{R}^{n}$, then the fractional type parabolic multilinear commutators generated by parabolic fractional maximal and integral operators with rough kernel and $\vec{b}=\left(b_{1}, \ldots, b_{m}\right)$ (parabolic local Campanato functions) are given as follows, respectively:

$$
\begin{gathered}
{\left[\vec{b}, T_{\Omega, \alpha}^{P}\right] f(x)=\int_{\mathbb{R}^{n}} \prod_{i=1}^{m}\left[b_{i}(x)-b_{i}(y)\right] \frac{\Omega(x-y)}{\rho(x-y)^{\gamma-\alpha}} f(y) d y, \quad 0<\alpha<\gamma,} \\
M_{\Omega, \vec{b}, \alpha}^{P} f(x)=\sup _{t>0}|E(x, t)|^{-1+\frac{\alpha}{\gamma}} \int_{E(x, t)} \prod_{i=1}^{m}\left[\left|b_{i}(x)-b_{i}(y)\right|\right]|\Omega(x-y)||f(y)| d y, \quad 0<\alpha<\gamma .
\end{gathered}
$$

We notice that when $\alpha=0$, the above operators become the parabolic multilinear commutators generated by parabolic singular integral operators and the corresponding parabolic maximal operators with rough kernel and $\vec{b}=\left(b_{1}, \ldots, b_{m}\right)$ as follows, respectively:

$$
\begin{gathered}
{\left[\vec{b}, T_{\Omega}^{P}\right] f(x)=\int_{\mathbb{R}^{n}} \prod_{i=1}^{m}\left[b_{i}(x)-b_{i}(y)\right] \frac{\Omega(x-y)}{\rho(x-y)^{\gamma}} f(y) d y,} \\
M_{\Omega, \vec{b}}^{P} f(x)=\sup _{t>0}|E(x, t)|^{-1} \int_{E(x, t)} \prod_{i=1}^{m}\left[\left|b_{i}(x)-b_{i}(y)\right|\right]|\Omega(x-y)||f(y)| d y .
\end{gathered}
$$

In $[7,8]$ the boundedness of a class of parabolic sublinear operators with rough kernel and their commutators on the parabolic generalized local Morrey spaces under generic size conditions which are satisfied by most of the operators in harmonic analysis has been investigated, respectively.

Inspired by $[7,8]$, our main purpose in this paper is to consider the boundedness of above operators $\left(\left[\vec{b}, T_{\Omega}\right], M_{\Omega, \vec{b}},\left[\vec{b}, T_{\Omega, \alpha}\right], M_{\Omega, \vec{b}, \alpha}\right)$ on the parabolic generalized local Morrey spaces, respectively. But, the techniques and non-trivial estimates which have been used in the proofs of our main results are quite different from $[7,8]$. For example, using inequality about the weighted Hardy operator $H_{w}$ in $[7,8]$, in this paper we will only use the following relationship between essential supremum and essential infimum

$$
(\underset{x \in E}{\operatorname{essinf}} f(x))^{-1}=\operatorname{esssup}_{x \in E} \frac{1}{f(x)},
$$

where $f$ is any real-valued nonnegative function and measurable on $E$ (see [13], page 143). Our main results can be formulated as follows. 
Theorem 1.3. Let $x_{0} \in \mathbb{R}^{n}, \Omega \in L_{s}\left(S^{n-1}\right), 1<s \leq \infty$, be $A_{t}$-homogeneous of degree zero. Let also, $1<q, p_{i}, p<\infty$ with $\frac{1}{q}=\sum_{i=1}^{m} \frac{1}{p_{i}}+\frac{1}{p}$ and $\vec{b} \in L C_{p_{i}, \lambda_{i}, P}^{\left\{x_{0}\right\}}\left(\mathbb{R}^{n}\right)$ for $0 \leq \lambda_{i}<\frac{1}{\gamma}$, $i=1, \ldots, m$.

For $s^{\prime} \leq q$, if functions $\varphi_{1}, \varphi_{2}: \mathbb{R}^{n} \times \mathbb{R}_{+} \rightarrow \mathbb{R}_{+}$satisfy the inequality

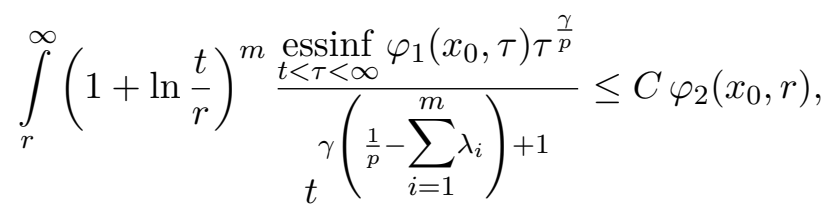

and for $p<s$, if functions $\varphi_{1}, \varphi_{2}: \mathbb{R}^{n} \times \mathbb{R}_{+} \rightarrow \mathbb{R}_{+}$satisfy the inequality

$$
\int_{r}^{\infty}\left(1+\ln \frac{t}{r}\right)^{m} \frac{\underset{\gamma}{\operatorname{essinf}} \varphi_{1}\left(x_{0}, \tau\right) \tau^{\frac{\gamma}{p}}}{t_{t}\left(\frac{1}{p}-\frac{1}{s}-\sum_{i=1}^{m} \lambda_{i}\right)+1} d t \leq C \varphi_{2}\left(x_{0}, r\right) r^{\frac{\gamma}{s}},
$$

where $C$ does not depend on $r$, then the commutators $\left[\vec{b}, T_{\Omega}^{P}\right]$ and $M_{\Omega, \vec{b}}^{P}$ are bounded from $L M_{p, \varphi_{1}, P}^{\left\{x_{0}\right\}}$ to $L M_{q, \varphi_{2}, P}^{\left\{x_{0}\right\}}$. Moreover,

$$
\begin{gathered}
\left\|\left[\vec{b}, T_{\Omega}^{P}\right] f\right\|_{L M_{q, \varphi_{2}, P}^{\left\{x_{0}\right\}}} \lesssim \prod_{i=1}^{m}\|\vec{b}\|_{L C_{p_{i}, \lambda_{i}, P}^{\left\{x_{0}\right\}}}\|f\|_{L M_{p, \varphi_{1}, P}^{\left\{x_{0}\right\}}}, \\
\left\|\left[\vec{b}, M_{\Omega, \vec{b}}^{P}\right] f\right\|_{L M_{q, \varphi_{2}, P}^{\left\{x_{0}\right\}}} \lesssim \prod_{i=1}^{m}\|\vec{b}\|_{L C_{p_{i}, \lambda_{i}, P}^{\left\{x_{0}\right\}}}\|f\|_{L M_{p, \varphi_{1}, P}^{\left\{x_{0}\right\}}} .
\end{gathered}
$$

Corollary 1.4 ([7]). Let $x_{0} \in \mathbb{R}^{n}, \Omega \in L_{s}\left(S^{n-1}\right), 1<s \leq \infty$, be $A_{t}$-homogeneous of degree zero. Let $T_{\Omega}^{P}$ be a parabolic linear operator satisfying condition (1.1), bounded on $L_{p}\left(\mathbb{R}^{n}\right)$ for $p>1$, and bounded from $L_{1}\left(\mathbb{R}^{n}\right)$ to $W L_{1}\left(\mathbb{R}^{n}\right)$. Let also, $b \in L C_{p_{2}, \lambda, P}^{\left\{x_{0}\right\}}\left(\mathbb{R}^{n}\right)$, $0 \leq \lambda<\frac{1}{\gamma}$ and $\frac{1}{p}=\frac{1}{p_{1}}+\frac{1}{p_{2}}$.

For $s^{\prime} \leq p$, if functions $\varphi_{1}, \varphi_{2}: \mathbb{R}^{n} \times \mathbb{R}_{+} \rightarrow \mathbb{R}_{+}$satisfy the inequality

$$
\int_{r}^{\infty}\left(1+\ln \frac{t}{r}\right) \frac{\operatorname{essinf}_{t<\tau<\infty} \varphi_{1}\left(x_{0}, \tau\right) \tau^{\frac{\gamma}{p_{1}}}}{t^{\frac{\gamma}{p_{1}}+1-\gamma \lambda}} d t \leq C \varphi_{2}\left(x_{0}, r\right),
$$

and for $p_{1}<s$, if functions $\varphi_{1}, \varphi_{2}: \mathbb{R}^{n} \times \mathbb{R}_{+} \rightarrow \mathbb{R}_{+}$satisfy the inequality

$$
\int_{r}^{\infty}\left(1+\ln \frac{t}{r}\right) \frac{\underset{t<\tau<\infty}{\operatorname{essinf}} \varphi_{1}\left(x_{0}, \tau\right) \tau^{\frac{\gamma}{p_{1}}}}{t^{\frac{\gamma}{p_{1}}-\frac{\gamma}{s}+1-\gamma \lambda}} d t \leq C \varphi_{2}\left(x_{0}, r\right) r^{\frac{\gamma}{s}},
$$

where $C$ does not depend on $r$, then the commutators $\left[b, T_{\Omega}^{P}\right]$ and $M_{\Omega, b}^{P}$ are bounded from $L M_{p_{1}, \varphi_{1}, P}^{\left\{x_{0}\right\}}$ to $L M_{p, \varphi_{2}, P}^{\left\{x_{0}\right\}}$. Moreover,

$$
\begin{gathered}
\left\|\left[b, T_{\Omega}^{P}\right] f\right\|_{L M_{p, \varphi_{2}, P}^{\left\{x_{0}\right\}}} \lesssim\|b\|_{L C_{p_{2}, \lambda, P}^{\left\{x_{0}\right\}}\|f\|_{L M_{p_{1}, \varphi_{1}, P}^{\left\{x_{0}\right\}}}}, \\
\left\|M_{\Omega, b}^{P} f\right\|_{L M_{p, \varphi_{2}, P}^{\left\{x_{0}\right\}}} \lesssim\|b\|_{L C_{p_{2}, \lambda, P}^{\left\{x_{0}\right\}}\|f\|_{L M_{p_{1}, \varphi_{1}, P}^{\left\{x_{0}\right\}}}} .
\end{gathered}
$$

Theorem 1.5. Let $x_{0} \in \mathbb{R}^{n}, \Omega \in L_{s}\left(S^{n-1}\right), 1<s \leq \infty$, be $A_{t}$-homogeneous of degree zero. Let also, $0<\alpha<\gamma$ and $1<q, q_{1}, p_{i}, p<\frac{\gamma}{\alpha}$ with $\frac{1}{q}=\sum_{i=1}^{m} \frac{1}{p_{i}}+\frac{1}{p}, \frac{1}{q_{1}}=\frac{1}{q}-\frac{\alpha}{\gamma}$ and $\vec{b} \in L C_{p_{i}, \lambda_{i}, P}^{\left\{x_{0}\right\}}\left(\mathbb{R}^{n}\right)$ for $0 \leq \lambda_{i}<\frac{1}{\gamma}, i=1, \ldots, m$. 
For $s^{\prime} \leq q$, if functions $\varphi_{1}, \varphi_{2}: \mathbb{R}^{n} \times \mathbb{R}_{+} \rightarrow \mathbb{R}_{+}$satisfy the inequality

$$
\int_{r}^{\infty}\left(1+\ln \frac{t}{r}\right)^{m} \frac{\underset{t<\tau<\infty}{\operatorname{essinf}} \varphi_{1}\left(x_{0}, \tau\right) \tau^{\frac{\gamma}{p}}}{\gamma\left(\frac{1}{q_{1}}-\left(\sum_{i=1}^{m} \lambda_{i}+\sum_{i=1}^{m} \frac{1}{p_{i}}\right)\right)+1} \leq C \varphi_{2}\left(x_{0}, r\right),
$$

and for $q_{1}<s$, if functions $\varphi_{1}, \varphi_{2}: \mathbb{R}^{n} \times \mathbb{R}_{+} \rightarrow \mathbb{R}_{+}$satisfy the inequality

$$
\int_{r}^{\infty}\left(1+\ln \frac{t}{r}\right)^{m} \frac{\operatorname{essinf}_{t<\tau<\infty} \varphi_{1}\left(x_{0}, \tau\right) \tau^{\frac{\gamma}{p}}}{\gamma\left(\frac{1}{q_{1}}-\left(\frac{1}{s}+\sum_{i=1}^{m} \lambda_{i}+\sum_{i=1}^{m} \frac{1}{p_{i}}\right)\right)+1} d t \leq C \varphi_{2}\left(x_{0}, r\right) r^{\frac{\gamma}{s}}
$$

where $C$ does not depend on $r$, then the commutators $\left[\vec{b}, T_{\Omega, \alpha}^{P}\right]$ and $M_{\Omega, \vec{b}, \alpha}^{P}$ are bounded from $L M_{p, \varphi_{1}, P}^{\left\{x_{0}\right\}}$ to $L M_{q_{1}, \varphi_{2}, P}^{\left\{x_{0}\right\}}$. Moreover,

$$
\begin{aligned}
&\left\|\left[\vec{b}, T_{\Omega, \alpha}^{P}\right] f\right\|_{L M_{q_{1}, \varphi_{2}, P}^{\left\{x_{0}\right\}}} \lesssim \prod_{i=1}^{m}\|\vec{b}\|_{L C_{p_{i}, \lambda_{i}, P}^{\left\{x_{0}\right\}}}\|f\|_{L M_{p, \varphi_{1}, P}^{\left\{x_{0}\right\}}}, \\
&\left\|M_{\Omega, \vec{b}, \alpha}^{P} f\right\|_{L M_{q_{1}, \varphi_{2}, P}^{\left\{x_{0}\right\}}} \lesssim \prod_{i=1}^{m}\|\vec{b}\|_{L C_{p_{i}, \lambda_{i}, P}^{\left\{x_{0}\right\}}}\|f\|_{L M_{p, \varphi_{1}, P}^{\left\{x_{0}\right\}}} .
\end{aligned}
$$

Corollary 1.6 ([8]). Let $x_{0} \in \mathbb{R}^{n}, \Omega \in L_{s}\left(S^{n-1}\right), 1<s \leq \infty$, be $A_{t}$-homogeneous of degree zero. Let $T_{\Omega, \alpha}^{P}$ be a parabolic linear operator satisfying condition (1.2) and bounded from $L_{p}\left(\mathbb{R}^{n}\right)$ to $L_{q}\left(\mathbb{R}^{n}\right)$. Let also, $0<\alpha<\gamma, 1<p<\frac{\gamma}{\alpha}, b \in L C_{p_{2}, \lambda, P}^{\left\{x_{0}\right\}}\left(\mathbb{R}^{n}\right), 0 \leq \lambda<\frac{1}{\gamma}$, $\frac{1}{p}=\frac{1}{p_{1}}+\frac{1}{p_{2}}, \frac{1}{q}=\frac{1}{p}-\frac{\alpha}{\gamma}, \frac{1}{q_{1}}=\frac{1}{p_{1}}-\frac{\alpha}{\gamma}$.

For $s^{\prime} \leq p$, if functions $\varphi_{1}, \varphi_{2}: \mathbb{R}^{n} \times \mathbb{R}_{+} \rightarrow \mathbb{R}_{+}$satisfy the inequality

$$
\int_{r}^{\infty}\left(1+\ln \frac{t}{r}\right) \frac{\underset{t<\tau<\infty}{\operatorname{essinf}} \varphi_{1}\left(x_{0}, \tau\right) \tau^{\frac{\gamma}{p_{1}}}}{t^{\frac{\gamma}{q_{1}}+1-\gamma \lambda}} d t \leq C \varphi_{2}\left(x_{0}, r\right),
$$

and for $q_{1}<s$, if functions $\varphi_{1}, \varphi_{2}: \mathbb{R}^{n} \times \mathbb{R}_{+} \rightarrow \mathbb{R}_{+}$satisfy the inequality

$$
\int_{r}^{\infty}\left(1+\ln \frac{t}{r}\right) \frac{\underset{t<\tau<\infty}{\operatorname{essinf}} \varphi_{1}\left(x_{0}, \tau\right) \tau^{\frac{\gamma}{p_{1}}}}{t^{\frac{\gamma}{q_{1}}-\frac{\gamma}{s}+1-\gamma \lambda}} d t \leq C \varphi_{2}\left(x_{0}, r\right) r^{\frac{\gamma}{s}},
$$

where $C$ does not depend on $r$, then the commutators $\left[b, T_{\Omega, \alpha}^{P}\right]$ and $M_{\Omega, b, \alpha}^{P}$ are bounded from $L M_{p_{1}, \varphi_{1}, P}^{\left\{x_{0}\right\}}$ to $L M_{q, \varphi_{2}, P}^{\left\{x_{0}\right\}}$. Moreover,

$$
\begin{aligned}
&\left\|\left[b, T_{\Omega, \alpha}^{P}\right] f\right\|_{L M_{q, \varphi_{2}, P}^{\left\{x_{0}\right\}}} \lesssim\|b\|_{L C_{p_{2}, \lambda, P}^{\left\{x_{0}\right\}}}\|f\|_{L M_{p_{1}, \varphi_{1}, P}^{\left\{x_{0}\right\}}}, \\
&\left\|M_{\Omega, b, \alpha}^{P} f\right\|_{L M_{q, \varphi_{2}, P}^{\left\{x_{0}\right\}}} \lesssim\|b\|_{L C_{p_{2}, \lambda, P}^{\left\{x_{0}\right\}}\|f\|_{L M_{p_{1}, \varphi_{1}, P}^{\left\{x_{0}\right\}}}} .
\end{aligned}
$$

At last, let $F, G \geq 0$. Here and henceforth, the symbol $F \approx G$ means that $F \lesssim G$ and $G \lesssim F$ happen simultaneously; while $F \lesssim G$ and $G \lesssim F$ mean that there exists a constant $C>0$ such that $F \leq C G$. 


\section{Some lemmas}

To prove the main results (Theorems 1.3 and 1.5), we need the following lemmas.

Lemma $2.1([7,8])$. Let $b$ be a parabolic local Campanato function in $L C_{p, \lambda, P}^{\left\{x_{0}\right\}}\left(\mathbb{R}^{n}\right), 1<$ $p<\infty, 0 \leq \lambda<\frac{1}{\gamma}$ and $0<r_{2}<r_{1}$. Then

$$
\left(\frac{1}{\left|E\left(x_{0}, r_{1}\right)\right|^{1+\lambda p}} \int_{E\left(x_{0}, r_{1}\right)}\left|b(y)-b_{E\left(x_{0}, r_{2}\right)}\right|^{p} d y\right)^{\frac{1}{p}} \leq C\left(1+\ln \frac{r_{1}}{r_{2}}\right)\|b\|_{L C_{p, \lambda, P}^{\left\{x_{0}\right\}}},
$$

where $C>0$ is independent of $b, r_{1}$ and $r_{2}$.

From this inequality (2.1), we have

$$
\left|b_{E\left(x_{0}, r_{1}\right)}-b_{E\left(x_{0}, r_{2}\right)}\right| \leq C\left(1+\ln \frac{r_{1}}{r_{2}}\right)\left|E\left(x_{0}, r_{1}\right)\right|^{\lambda}\|b\|_{L C_{p, \lambda, P}^{\left\{x_{0}\right\}}},
$$

and it is easy to see that

$$
\left\|b-b_{E}\right\|_{L_{p}(E)} \leq C\left(1+\ln \frac{r_{1}}{r_{2}}\right) r^{\frac{\gamma}{p}+\gamma \lambda}\|b\|_{L C_{p, \lambda, P}^{\left\{x_{0}\right\}}} .
$$

Lemma 2.2. Suppose that $x_{0} \in \mathbb{R}^{n}, \Omega \in L_{s}\left(S^{n-1}\right), 1<s \leq \infty$, is $A_{t}$-homogeneous of degree zero. Let $T_{\Omega}^{P}$ be a parabolic linear operator satisfying condition (1.1). Let also $1<q, p_{i}, p<\infty$ with $\frac{1}{q}=\sum_{i=1}^{m} \frac{1}{p_{i}}+\frac{1}{p}$ and $\vec{b} \in L C_{p_{i}, \lambda_{i}, P}^{\left\{x_{0}\right\}}\left(\mathbb{R}^{n}\right)$ for $0 \leq \lambda_{i}<\frac{1}{\gamma}, i=1, \ldots, m$. Then, for $s^{\prime} \leq q$ the inequality

$$
\left\|\left[\vec{b}, T_{\Omega}^{P}\right] f\right\|_{L_{q}\left(E\left(x_{0}, r\right)\right)} \lesssim \prod_{i=1}^{m}\|\vec{b}\|_{L C_{p_{i}, \lambda_{i}, P}^{\left\{x_{0}\right\}}} r^{\frac{\gamma}{q}} \int_{2 k r}^{\infty}\left(1+\ln \frac{t}{r}\right)^{m} \frac{\|f\|_{L_{p}\left(E\left(x_{0}, t\right)\right)}}{{ }_{t}\left(\frac{1}{p}-\sum_{i=1}^{m} \lambda_{i}\right)+1} d t
$$

holds for any ellipsoid $E\left(x_{0}, r\right)$ and for all $f \in L_{p}^{\text {loc }}\left(\mathbb{R}^{n}\right)$. Also, for $p<s$ the inequality

$$
\left\|\left[\vec{b}, T_{\Omega}^{P}\right] f\right\|_{L_{q}\left(E\left(x_{0}, r\right)\right)} \lesssim \prod_{i=1}^{m}\|\vec{b}\|_{L C_{p_{i}, \lambda_{i}, P}^{\left\{x_{0}\right\}}} r^{\frac{\gamma}{q}-\frac{\gamma}{s}} \int_{2 k r}^{\infty}\left(1+\ln \frac{t}{r}\right)^{m} \frac{\|f\|_{L_{p}\left(E\left(x_{0}, t\right)\right)}}{\gamma\left(\frac{1}{p}-\frac{1}{s}-\sum_{i=1}^{m} \lambda_{i}\right)+1} d t
$$

holds for any ellipsoid $E\left(x_{0}, r\right)$ and for all $f \in L_{p}^{l o c}\left(\mathbb{R}^{n}\right)$.

Proof. Without loss of generality, it is sufficient to show that the conclusion holds for $\left[\vec{b}, T_{\Omega}^{P}\right] f=\left[\left(b_{1}, b_{2}\right), T_{\Omega}^{P}\right] f$. Let $1<q, p_{i}, p<\infty$ with $\frac{1}{q}=\sum_{i=1}^{m} \frac{1}{p_{i}}+\frac{1}{p}$ and $\vec{b} \in L C_{p_{i}, \lambda_{i}, P}^{\left\{x_{0}\right\}}\left(\mathbb{R}^{n}\right)$ for $0 \leq \lambda_{i}<\frac{1}{\gamma}, i=1, \ldots, m$. Set $E=E\left(x_{0}, r\right)$ for the parabolic ball (ellipsoid) centered at $x_{0}$ and of radius $r$ and for $k>0$, we denote $2 k E=E\left(x_{0}, 2 k r\right)=\left\{y \in \mathbb{R}^{n}: \rho(x-y)<2 k r\right\}$. We represent $f$ as

$$
f=f_{1}+f_{2}, \quad f_{1}(y)=f(y) \chi_{2 k E}(y), \quad f_{2}(y)=f(y) \chi_{(2 k E)^{C}}(y), \quad r>0
$$

and thus have

$$
\left\|\left[\left(b_{1}, b_{2}\right), T_{\Omega}^{P}\right] f\right\|_{L_{q}(E)} \leq\left\|\left[\left(b_{1}, b_{2}\right), T_{\Omega}^{P}\right] f_{1}\right\|_{L_{q}(E)}+\left\|\left[\left(b_{1}, b_{2}\right), T_{\Omega}^{P}\right] f_{2}\right\|_{L_{q}(E)}=: F+G .
$$

Let us estimate $F+G$, respectively. 
For $\left[\left(b_{1}, b_{2}\right), T_{\Omega}^{P}\right] f_{1}(x)$, we have the following decomposition,

$$
\begin{aligned}
{\left[\left(b_{1}, b_{2}\right), T_{\Omega}^{P}\right] f_{1}(x)=} & \left(b_{1}(x)-\left(b_{1}\right)_{E}\right)\left(b_{2}(x)-\left(b_{2}\right)_{E}\right) T_{\Omega}^{P} f_{1}(x) \\
& -\left(b_{1}(\cdot)-\left(b_{1}\right)_{E}\right) T_{\Omega}^{P}\left(\left(b_{2}(\cdot)-\left(b_{2}\right)_{E}\right) f_{1}\right)(x) \\
& +\left(b_{2}(x)-\left(b_{2}\right)_{E}\right) T_{\Omega}^{P}\left(\left(b_{1}(x)-\left(b_{1}\right)_{E}\right) f_{1}\right)(x) \\
& -T_{\Omega}^{P}\left(\left(b_{1}(\cdot)-\left(b_{1}\right)_{E}\right)\left(b_{2}(\cdot)-\left(b_{2}\right)_{E}\right) f_{1}\right)(x) .
\end{aligned}
$$

Hence, we get

$$
\begin{aligned}
F=\left\|\left[\left(b_{1}, b_{2}\right), T_{\Omega}^{P}\right] f_{1}\right\|_{L_{q}(E)} \lesssim & \left\|\left(b_{1}-\left(b_{1}\right)_{E}\right)\left(b_{2}(x)-\left(b_{2}\right)_{E}\right) T_{\Omega}^{P} f_{1}\right\|_{L_{q}(E)} \\
& +\left\|\left(b_{1}-\left(b_{1}\right)_{E}\right) T_{\Omega}^{P}\left(\left(b_{2}-\left(b_{2}\right)_{E}\right) f_{1}\right)\right\|_{L_{q}(E)} \\
& +\left\|\left(b_{2}-\left(b_{2}\right)_{E}\right) T_{\Omega}^{P}\left(\left(b_{1}-\left(b_{1}\right)_{E}\right) f_{1}\right)\right\|_{L_{q}(E)} \\
& +\left\|T_{\Omega}^{P}\left(\left(b_{1}-\left(b_{1}\right)_{E}\right)\left(b_{2}-\left(b_{2}\right)_{E}\right) f_{1}\right)\right\|_{L_{q}(E)} \\
\equiv & F_{1}+F_{2}+F_{3}+F_{4} .
\end{aligned}
$$

One observes that the estimate of $F_{2}$ is analogous to that of $F_{3}$. Thus, we will only estimate $F_{1}, F_{2}$ and $F_{4}$.

To estimate $F_{1}$, let $1<q, \tau<\infty$, such that $\frac{1}{q}=\frac{1}{\tau}+\frac{1}{p}, \frac{1}{\tau}=\frac{1}{p_{1}}+\frac{1}{p_{2}}$. Then, using Hölder's inequality and by Theorem 1.2. in [7] it follows that:

$$
\begin{aligned}
F_{1} & =\left\|\left(b_{1}-\left(b_{1}\right)_{E}\right)\left(b_{2}(x)-\left(b_{2}\right)_{E}\right) T_{\Omega}^{P} f_{1}\right\|_{L_{q}(E)} \\
& \lesssim\left\|\left(b_{1}-\left(b_{1}\right)_{E}\right)\left(b_{2}(x)-\left(b_{2}\right)_{E}\right)\right\|_{L_{\tau}(E)}\left\|T_{\Omega}^{P} f_{1}\right\|_{L_{p}(E)} \\
& \lesssim\left\|b_{1}-\left(b_{1}\right)_{E}\right\|_{L_{p_{1}}(E)}\left\|b_{2}-\left(b_{2}\right)_{E}\right\|_{L_{p_{2}}(E)}\|f\|_{L_{p}(2 k E)} \\
& \lesssim\left\|b_{1}-\left(b_{1}\right)_{E}\right\|_{L_{p_{1}}(E)}\left\|b_{2}-\left(b_{2}\right)_{E}\right\|_{L_{p_{2}}(E)} r^{\frac{\gamma}{p}} \int_{2 k r}^{\infty}\|f\|_{L_{p}\left(E\left(x_{0}, t\right)\right)} \frac{d t}{t^{\frac{\gamma}{p}+1}}
\end{aligned}
$$

From Lemma 2.1, it is easy to see that

$$
\left\|b_{i}-\left(b_{i}\right)_{E}\right\|_{L_{p_{i}}(E)} \leq C r^{\frac{\gamma}{p_{i}}+\gamma \lambda_{i}}\left\|b_{i}\right\|_{L C_{p_{i}, \lambda_{i}, P}^{\left\{x_{0}\right\}}},
$$

and

$$
\begin{aligned}
\left\|b_{i}-\left(b_{i}\right)_{E}\right\|_{L_{p_{i}}(2 k E)} & \leq\left\|b_{i}-\left(b_{i}\right)_{2 k E}\right\|_{L_{p_{i}}(2 k E)}+\left\|\left(b_{i}\right)_{E}-\left(b_{i}\right)_{2 k E}\right\|_{L_{p_{i}}(2 k E)} \\
& \lesssim r^{\frac{\gamma}{p_{i}}+\gamma \lambda_{i}}\left\|b_{i}\right\|_{L C_{p_{i}, \lambda_{i}, P}^{\left\{x_{0}\right\}}}
\end{aligned}
$$

for $i=1,2$. Hence, by (2.7) we get

$$
\begin{aligned}
F_{1} & \lesssim\left\|b_{1}\right\|_{L C_{p_{1}, \lambda_{1}, P}^{\left\{x_{0}\right\}}}\left\|b_{2}\right\|_{L C_{p_{2}, \lambda_{2}, P}^{\left\{x_{0}\right\}}} r^{\gamma\left(\frac{1}{p_{1}}+\frac{1}{p_{2}}+\frac{1}{p}\right)} \int_{2 k r}^{\infty}\left(1+\ln \frac{t}{r}\right)^{2} t^{-\frac{\gamma}{p}+\gamma\left(\lambda_{1}+\lambda_{2}\right)-1}\|f\|_{L_{p}\left(E\left(x_{0}, t\right)\right)} d t \\
& \lesssim\left\|b_{1}\right\|_{L C_{p_{1}, \lambda_{1}, P}^{\left\{x_{0}\right\}}}\left\|b_{2}\right\|_{L C_{p_{2}, \lambda_{2}, P}^{\left\{x_{0}\right\}}} r^{\frac{\gamma}{q}} \int_{2 k r}^{\infty}\left(1+\ln \frac{t}{r}\right)^{2} \frac{\|f\|_{L_{p}\left(E\left(x_{0}, t\right)\right)}}{t^{\frac{\gamma}{p}-\gamma\left(\lambda_{1}+\lambda_{2}\right)+1}} d t .
\end{aligned}
$$


To estimate $F_{2}$, let $1<\tau<\infty$, such that $\frac{1}{q}=\frac{1}{p_{1}}+\frac{1}{\tau}$. Then, similar to the estimates for $F_{1}$, we have

$$
\begin{aligned}
F_{2} & \lesssim\left\|b_{1}-\left(b_{1}\right)_{E}\right\|_{L_{p_{1}}(E)}\left\|T_{\Omega}^{P}\left(\left(b_{2}(\cdot)-\left(b_{2}\right)_{E}\right) f_{1}\right)\right\|_{L_{\tau}(E)} \\
& \lesssim\left\|b_{1}-\left(b_{1}\right)_{E}\right\|_{L_{p_{1}}(E)}\left\|\left(b_{2}(\cdot)-\left(b_{2}\right)_{E}\right) f_{1}\right\|_{L_{k}(E)} \\
& \lesssim\left\|b_{1}-\left(b_{1}\right)_{E}\right\|_{L_{p_{1}}(E)}\left\|b_{2}-\left(b_{2}\right)_{E}\right\|_{L_{p_{2}}(2 k E)}\|f\|_{L_{p}(2 k E)},
\end{aligned}
$$

where $1<k<\infty$, such that $\frac{1}{k}=\frac{1}{p_{2}}+\frac{1}{p}=\frac{1}{\tau}$. By (2.7) and (2.8), we get

$$
F_{2} \lesssim\left\|b_{1}\right\|_{L C_{p_{1}, \lambda_{1}, P}^{\left\{x_{0}\right\}}}\left\|b_{2}\right\|_{L C_{p_{2}, \lambda_{2}, P}^{\left\{x_{0}\right\}}} r^{\frac{\gamma}{q}} \int_{2 k r}^{\infty}\left(1+\ln \frac{t}{r}\right)^{2} \frac{\|f\|_{L_{p}\left(E\left(x_{0}, t\right)\right)}}{t^{\frac{\gamma}{p}-\gamma\left(\lambda_{1}+\lambda_{2}\right)+1}} d t .
$$

In a similar way, $F_{3}$ has the same estimate as above, so we omit the details. Then we have that

$$
F_{3} \lesssim\left\|b_{1}\right\|_{L C_{p_{1}, \lambda_{1}, P}^{\left\{x_{0}\right\}}}\left\|b_{2}\right\|_{L C_{p_{2}, \lambda_{2}, P}^{\left\{x_{0}\right\}}} r^{\frac{\gamma}{q}} \int_{2 k r}^{\infty}\left(1+\ln \frac{t}{r}\right)^{2} \frac{\|f\|_{L_{p}\left(E\left(x_{0}, t\right)\right)}}{t^{\frac{\gamma}{p}-\gamma\left(\lambda_{1}+\lambda_{2}\right)+1}} d t .
$$

Now let us consider the term $F_{4}$. Let $1<q, \tau<\infty$, such that $\frac{1}{q}=\frac{1}{\tau}+\frac{1}{p}, \frac{1}{\tau}=\frac{1}{p_{1}}+\frac{1}{p_{2}}$. Then by Theorem 1.2. in [7], Hölder's inequality and (2.8), we obtain

$$
\begin{aligned}
F_{4} & =\left\|T_{\Omega}^{P}\left(\left(b_{1}-\left(b_{1}\right)_{E}\right)\left(b_{2}-\left(b_{2}\right)_{E}\right) f_{1}\right)\right\|_{L_{q}(E)} \\
& \lesssim\left\|\left(b_{1}-\left(b_{1}\right)_{E}\right)\left(b_{2}-\left(b_{2}\right)_{E}\right) f_{1}\right\|_{L_{q}(E)} \\
& \lesssim\left\|\left(b_{1}-\left(b_{1}\right)_{E}\right)\left(b_{2}-\left(b_{2}\right)_{E}\right)\right\|_{L_{\tau}(E)}\left\|f_{1}\right\|_{L_{p}(E)} \\
& \lesssim\left\|b_{1}-\left(b_{1}\right)_{E}\right\|_{L_{p_{1}}(2 k E)}\left\|b_{2}-\left(b_{2}\right)_{E}\right\|_{L_{p_{2}}(2 k E)}\|f\|_{L_{p}(2 k E)} \\
& \lesssim\left\|b_{1}\right\|_{L C_{p_{1}, \lambda_{1}, P}^{\left\{x_{0}\right\}}}\left\|b_{2}\right\|_{L C_{p_{2}, \lambda_{2}, P}^{\left\{x_{0}\right\}}} r^{\frac{\gamma}{q}} \int_{2 k r}^{\infty}\left(1+\ln \frac{t}{r}\right)^{2} \frac{\|f\|_{L_{p}\left(E\left(x_{0}, t\right)\right)}}{t^{\frac{\gamma}{p}-\gamma\left(\lambda_{1}+\lambda_{2}\right)+1}} d t .
\end{aligned}
$$

Combining all the estimates of $F_{1}, F_{2}, F_{3}, F_{4}$; we get

$$
F=\left\|\left[\left(b_{1}, b_{2}\right), T_{\Omega}^{P}\right] f_{1}\right\|_{L_{q}(E)} \lesssim\left\|b_{1}\right\|_{L C_{p_{1}, \lambda_{1}, P}^{\left\{x_{0}\right\}}}\left\|b_{2}\right\|_{L C_{p_{2}, \lambda_{2}, P}^{\left\{x_{0}\right\}}} r^{\frac{\gamma}{q}} \int_{2 k r}^{\infty}\left(1+\ln \frac{t}{r}\right)^{2} \frac{\|f\|_{L_{p}\left(E\left(x_{0}, t\right)\right)}}{t^{\frac{\gamma}{p}-\gamma\left(\lambda_{1}+\lambda_{2}\right)+1}} d t .
$$

Now, let us estimate $G=\left\|\left[\left(b_{1}, b_{2}\right), T_{\Omega}^{P}\right] f_{2}\right\|_{L_{q}(E)}$. For $G$, it's similar to (2.6) we also write

$$
\begin{aligned}
G=\left\|\left[\left(b_{1}, b_{2}\right), T_{\Omega}^{P}\right] f_{2}\right\|_{L_{q}(E)} \lesssim & \left\|\left(b_{1}-\left(b_{1}\right)_{E}\right)\left(b_{2}(x)-\left(b_{2}\right)_{E}\right) T_{\Omega}^{P} f_{2}\right\|_{L_{q}(E)} \\
& +\left\|\left(b_{1}-\left(b_{1}\right)_{E}\right) T_{\Omega}^{P}\left(\left(b_{2}-\left(b_{2}\right)_{E}\right) f_{2}\right)\right\|_{L_{q}(E)} \\
& +\left\|\left(b_{2}-\left(b_{2}\right)_{E}\right) T_{\Omega}^{P}\left(\left(b_{1}-\left(b_{1}\right)_{E}\right) f_{2}\right)\right\|_{L_{q}(E)} \\
& +\left\|T_{\Omega}^{P}\left(\left(b_{1}-\left(b_{1}\right)_{E}\right)\left(b_{2}-\left(b_{2}\right)_{E}\right) f_{2}\right)\right\|_{L_{q}(E)} \\
\equiv & G_{1}+G_{2}+G_{3}+G_{4} .
\end{aligned}
$$


To estimate $G_{1}$, let $1<q, \tau<\infty$, such that $\frac{1}{q}=\frac{1}{\tau}+\frac{1}{p}, \frac{1}{\tau}=\frac{1}{p_{1}}+\frac{1}{p_{2}}$. Then, using Hölder's inequality and by (12) in [7] and (2.7), we have

$$
\begin{aligned}
& G_{1}=\left\|\left(b_{1}-\left(b_{1}\right)_{E}\right)\left(b_{2}(x)-\left(b_{2}\right)_{E}\right) T_{\Omega}^{P} f_{2}\right\|_{L_{q}(E)} \\
& \lesssim\left\|\left(b_{1}-\left(b_{1}\right)_{E}\right)\left(b_{2}-\left(b_{2}\right)_{E}\right)\right\|_{L_{\tau}(E)}\left\|T_{\Omega}^{P} f_{2}\right\|_{L_{p}(E)} \\
& \lesssim\left\|b_{1}-\left(b_{1}\right)_{E}\right\|_{L_{p_{1}}(E)}\left\|b_{2}-\left(b_{2}\right)_{E}\right\|_{L_{p_{2}}(E)} r^{\frac{\gamma}{p}} \int_{2 k r}^{\infty}\|f\|_{L_{p}\left(E\left(x_{0}, t\right) t^{-\frac{\gamma}{p}-1} d t\right.} \\
& \lesssim\left\|b_{1}\right\|_{L C_{p_{1}, \lambda_{1}, P}^{\left\{x_{0}\right\}}}\left\|b_{2}\right\|_{L C_{p_{2}, \lambda_{2}, P}^{\left\{x_{0}\right\}}} r^{\gamma\left(\frac{1}{p_{1}}+\frac{1}{p_{2}}+\frac{1}{p}\right)} \\
& \times \int_{2 k r}^{\infty}\left(1+\ln \frac{t}{r}\right)^{2} t^{-\frac{\gamma}{p}+\gamma\left(\lambda_{1}+\lambda_{2}\right)-1}\|f\|_{L_{p}\left(E\left(x_{0}, t\right)\right)} d t \\
& \lesssim\left\|b_{1}\right\|_{L C_{p_{1}, \lambda_{1}, P}^{\left\{x_{0}\right\}}}\left\|b_{2}\right\|_{L C_{p_{2}, \lambda_{2}, P}^{\left\{x_{0}\right\}}} r^{\frac{\gamma}{q}} \int_{2 k r}^{\infty}\left(1+\ln \frac{t}{r}\right)^{2} \frac{\|f\|_{L_{p}\left(E\left(x_{0}, t\right)\right)}}{t^{\frac{\gamma}{p}-\gamma\left(\lambda_{1}+\lambda_{2}\right)+1}} d t .
\end{aligned}
$$

On the other hand, for the estimates used in $G_{2}, G_{3}$, we have to prove the below inequality:

$$
\left|T_{\Omega}^{P}\left(\left(b_{2}-\left(b_{2}\right)_{E}\right) f_{2}\right)(x)\right| \lesssim\left\|b_{2}\right\|_{L C_{p_{2}, \lambda_{2}, P}^{\left\{x_{0}\right\}}} \int_{2 k r}^{\infty}\left(1+\ln \frac{t}{r}\right) t^{-\frac{\gamma}{p}+\gamma \lambda_{2}-1}\|f\|_{L_{p}\left(E\left(x_{0}, t\right)\right)} d t .
$$

Indeed, when $s^{\prime} \leq q$, for $x \in E$, by Fubini's theorem and applying Hölder's inequality and from (2.2), (2.3), $0<r<t,(11)$ in [7] we have

$$
\begin{aligned}
\mid T_{\Omega}^{P} & \left(\left(b_{2}(\cdot)-\left(b_{2}\right)_{E}\right) f_{2}\right)(x) \mid \\
& \lesssim \int_{(2 k E)^{C}}\left|b_{2}(y)-\left(b_{2}\right)_{E}\right||\Omega(x-y)| \frac{|f(y)|}{\rho\left(x_{0}-y\right)^{\gamma}} d y \\
& \approx \int_{2 k r 2 k r<\rho\left(x_{0}-y\right)<t}^{\infty}\left|b_{2}(y)-\left(b_{2}\right)_{E}\right||\Omega(x-y)||f(y)| d y \frac{d t}{t^{\gamma+1}} \\
& \lesssim \int_{2 k r E\left(x_{0}, t\right)}^{\infty}\left|b_{2}(y)-\left(b_{2}\right)_{E\left(x_{0}, t\right)}\right||\Omega(x-y)||f(y)| d y \frac{d t}{t^{\gamma+1}} \\
& +\int_{2 k r}^{\infty}\left|\left(b_{2}\right)_{E\left(x_{0}, t\right)}-\left(b_{2}\right)_{E\left(x_{0}, r\right)}\right| \int_{E\left(x_{0}, t\right)}|\Omega(x-y)||f(y)| d y \frac{d t}{t^{\gamma+1}} \\
& \lesssim \int_{2 k r}^{\infty}\left\|b_{2}(\cdot)-\left.\left(b_{2}\right)_{E\left(x_{0}, t\right)}\right|_{L_{p_{2}}\left(E\left(x_{0}, t\right)\right)}\right\| \Omega(\cdot-y)\left\|_{L_{s}\left(E\left(x_{0}, t\right)\right)}\right\| f \|_{L_{p}\left(E\left(x_{0}, t\right)\right)} \\
& \times\left|E\left(x_{0}, t\right)\right|^{1-\frac{1}{p_{2}}-\frac{1}{s}-\frac{1}{p} \frac{d t}{t^{\gamma+1}}} \\
& +\int_{2 k r}^{\infty}\left|\left(b_{2}\right)_{E\left(x_{0}, t\right)}-\left(b_{2}\right)_{E\left(x_{0}, r\right)}\right|\|f\|_{L_{p}\left(E\left(x_{0}, t\right)\right)}\|\Omega(\cdot-y)\|_{L_{s}\left(E\left(x_{0}, t\right)\right)}\left|E\left(x_{0}, t\right)\right|^{1-\frac{1}{p}-\frac{1}{s}} \frac{d t}{t^{\gamma+1}} \\
& \quad \int_{2 k r}^{\infty}\left\|b_{2}(\cdot)-\left(b_{2}\right)_{E\left(x_{0}, t\right)}\right\|_{L_{p_{2}}\left(E\left(x_{0}, t\right)\right)}\|f\|_{L_{p}\left(E\left(x_{0}, t\right)\right)} t^{-1-\frac{\gamma}{p_{2}}-\frac{\gamma}{p}} d t \\
&
\end{aligned}
$$




$$
\begin{aligned}
&+\left\|b_{2}\right\|_{L C_{p_{2}, \lambda_{2}, P}\left\{x_{0}\right\}} \int_{2 k r}^{\infty}\left(1+\ln \frac{t}{r}\right)\|f\|_{L_{p}\left(E\left(x_{0}, t\right)\right)} t^{-1-\frac{\gamma}{p}+\gamma \lambda_{2}} d t \\
& \lesssim\left\|b_{2}\right\|_{L C_{p_{2}, \lambda_{2}, P}^{\left\{x_{0}\right\}}} \int_{2 k r}^{\infty}\left(1+\ln \frac{t}{r}\right) t^{-\frac{\gamma}{p}+\gamma \lambda_{2}-1}\|f\|_{L_{p}\left(E\left(x_{0}, t\right)\right)} d t .
\end{aligned}
$$

This completes the proof of inequality (2.9).

Let $1<\tau<\infty$, such that $\frac{1}{q}=\frac{1}{p_{1}}+\frac{1}{\tau}$. Then, using Hölder's inequality and from (2.9), (2.3) and $0<r<t$, we get

$$
\begin{aligned}
G_{2} & =\left\|\left(b_{1}-\left(b_{1}\right)_{E}\right) T_{\Omega}^{P}\left(\left(b_{2}-\left(b_{2}\right)_{E}\right) f_{2}\right)\right\|_{L_{q}(E)} \\
& \lesssim\left\|b_{1}-\left(b_{1}\right)_{E}\right\|_{L_{p_{1}}(E)}\left\|T_{\Omega}^{P}\left(\left(b_{2}(\cdot)-\left(b_{2}\right)_{E}\right) f_{2}\right)\right\|_{L_{\tau}(E)} \\
& \lesssim\left\|b_{1}\right\|_{L C_{p_{1}, \lambda_{1}, P}^{\left\{x_{0}\right\}}}\left\|b_{2}\right\|_{L C_{p_{2}, \lambda_{2}, P}^{\left\{x_{0}\right\}}} r^{\frac{\gamma}{q}} \int_{2 k r}^{\infty}\left(1+\ln \frac{t}{r}\right)^{2} \frac{\|f\|_{L_{p}\left(E\left(x_{0}, t\right)\right)}}{t^{\frac{\gamma}{p}-\gamma\left(\lambda_{1}+\lambda_{2}\right)+1}} d t .
\end{aligned}
$$

Similarly, $G_{3}$ has the same estimate above, so here we omit the details. Then the inequality

$$
\begin{aligned}
G_{3} & =\left\|\left(b_{2}-\left(b_{2}\right)_{E}\right) T_{\Omega}^{P}\left(\left(b_{1}-\left(b_{1}\right)_{E}\right) f_{2}\right)\right\|_{L_{q}(E)} \\
& \lesssim\left\|b_{1}\right\|_{L C_{p_{1}, \lambda_{1}, P}^{\left\{x_{0}\right\}}}\left\|b_{2}\right\|_{L C_{p_{2}, \lambda_{2}, P}^{\left\{x_{0}\right\}}} r^{\frac{\gamma}{q}} \int_{2 k r}^{\infty}\left(1+\ln \frac{t}{r}\right)^{2} \frac{\|f\|_{L_{p}\left(E\left(x_{0}, t\right)\right)}}{t^{\frac{\gamma}{p}-\gamma\left(\lambda_{1}+\lambda_{2}\right)+1}} d t
\end{aligned}
$$

is valid.

Now, let us estimate $G_{4}=\left\|T_{\Omega}^{P}\left(\left(b_{1}-\left(b_{1}\right)_{E}\right)\left(b_{2}-\left(b_{2}\right)_{E}\right) f_{2}\right)\right\|_{L_{q}(E)}$. It's similar to the estimate of (2.9), for any $x \in E$, we also write

$$
\begin{aligned}
& \left|T_{\Omega}^{P}\left(\left(b_{1}-\left(b_{1}\right)_{E}\right)\left(b_{2}-\left(b_{2}\right)_{E}\right) f_{2}\right)(x)\right| \\
& \quad \lesssim \int_{2 k r E\left(x_{0}, t\right)}^{\infty}\left|b_{1}(y)-\left(b_{1}\right)_{E\left(x_{0}, t\right)}\right|\left|b_{2}(y)-\left(b_{2}\right)_{E\left(x_{0}, t\right)}\right||\Omega(x-y)||f(y)| d y \frac{d t}{t^{\gamma+1}} \\
& \quad+\int_{2 k r E\left(x_{0}, t\right)}^{\infty}\left|b_{1}(y)-\left(b_{1}\right)_{E\left(x_{0}, t\right)}\right|\left|\left(b_{2}\right)_{E\left(x_{0}, t\right)}-\left(b_{2}\right)_{E\left(x_{0}, r\right)}\right||\Omega(x-y)||f(y)| d y \frac{d t}{t^{\gamma+1}} \\
& \quad+\int_{2 k r E\left(x_{0}, t\right)}^{\infty}\left|\left(b_{1}\right)_{E\left(x_{0}, t\right)}-\left(b_{2}\right)_{E\left(x_{0}, r\right)}\right|\left|b_{2}(y)-\left(b_{2}\right)_{E\left(x_{0}, t\right)}\right||\Omega(x-y)||f(y)| d y \frac{d t}{t^{\gamma+1}} \\
& \quad+\int_{2 k r E\left(x_{0}, t\right)}^{\infty}\left|\left(b_{1}\right)_{E\left(x_{0}, t\right)}-\left(b_{2}\right)_{E\left(x_{0}, r\right)}\right|\left|\left(b_{2}\right)_{E\left(x_{0}, t\right)}-\left(b_{2}\right)_{E\left(x_{0}, r\right)}\right| \Omega(x-y)|| f(y) \mid d y \frac{d t}{t^{\gamma+1}} \\
& \quad \equiv G_{41}+G_{42}+G_{43}+G_{44} .
\end{aligned}
$$

Let us estimate $G_{41}, G_{42}, G_{43}, G_{44}$, respectively.

Firstly, to estimate $G_{41}$, similar to the estimate of (2.9), we get

$$
G_{41} \lesssim\left\|b_{1}\right\|_{L C_{p_{1}, \lambda_{1}, P}^{\left\{x_{0}\right\}}}\left\|b_{2}\right\|_{L C_{p_{2}, \lambda_{2}, P}^{\left\{x_{0}\right\}}} \int_{2 k r}^{\infty}\left(1+\ln \frac{t}{r}\right)^{2} \frac{\|f\|_{L_{p}\left(E\left(x_{0}, t\right)\right)}}{t^{\frac{\gamma}{p}-\gamma\left(\lambda_{1}+\lambda_{2}\right)+1}} d t .
$$

Secondly, to estimate $G_{42}$ and $G_{43}$, from (2.9), (2.2), (2.3) and $0<r<t$, it follows that

$$
G_{42} \lesssim\left\|b_{1}\right\|_{L C_{p_{1}, \lambda_{1}, P}^{\left\{x_{0}\right\}}}\left\|b_{2}\right\|_{L C_{p_{2}, \lambda_{2}, P}^{\left\{x_{0}\right\}}} \int_{2 k r}^{\infty}\left(1+\ln \frac{t}{r}\right)^{2} \frac{\|f\|_{L_{p}\left(E\left(x_{0}, t\right)\right)}}{t^{\frac{\gamma}{p}-\gamma\left(\lambda_{1}+\lambda_{2}\right)+1}} d t
$$


and

$$
G_{43} \lesssim\left\|b_{1}\right\|_{L C_{p_{1}, \lambda_{1}, P}^{\left\{x_{0}\right\}}}\left\|b_{2}\right\|_{L C_{p_{2}, \lambda_{2}, P}^{\left\{x_{0}\right\}}} \int_{2 k r}^{\infty}\left(1+\ln \frac{t}{r}\right)^{2} \frac{\|f\|_{L_{p}\left(E\left(x_{0}, t\right)\right)}}{t^{\frac{\gamma}{p}-\gamma\left(\lambda_{1}+\lambda_{2}\right)+1}} d t
$$

Finally, to estimate $G_{44}$, similar to the estimate of (2.9), from (2.2), (2.3) and $0<r<t$, we have

$$
G_{44} \lesssim\left\|b_{1}\right\|_{L C_{p_{1}, \lambda_{1}, P}^{\left\{x_{0}\right\}}}\left\|b_{2}\right\|_{L C_{p_{2}, \lambda_{2}, P}^{\left\{x_{0}\right\}}} \int_{2 k r}^{\infty}\left(1+\ln \frac{t}{r}\right)^{2} \frac{\|f\|_{L_{p}\left(E\left(x_{0}, t\right)\right)}}{t^{\frac{\gamma}{p}-\gamma\left(\lambda_{1}+\lambda_{2}\right)+1}} d t
$$

By the estimates of $G_{4 j}$ above, where $j=1,2,3$, we know that

$$
\begin{aligned}
& \left|T_{\Omega}^{P}\left(\left(b_{1}-\left(b_{1}\right)_{E}\right)\left(b_{2}-\left(b_{2}\right)_{E}\right) f_{2}\right)(x)\right| \\
& \quad \lesssim\left\|b_{1}\right\|_{L C_{p_{1}, \lambda_{1}, P}^{\left\{x_{0}\right\}}}\left\|b_{2}\right\|_{L C_{p_{2}, \lambda_{2}, P}^{\left\{x_{0}\right\}}} \int_{2 k r}^{\infty}\left(1+\ln \frac{t}{r}\right)^{2} \frac{\|f\|_{L_{p}\left(E\left(x_{0}, t\right)\right)}}{t^{\frac{\gamma}{p}-\gamma\left(\lambda_{1}+\lambda_{2}\right)+1}} d t .
\end{aligned}
$$

Then, we have

$$
\begin{aligned}
G_{4} & =\left\|T_{\Omega}^{P}\left(\left(b_{1}-\left(b_{1}\right)_{E}\right)\left(b_{2}-\left(b_{2}\right)_{E}\right) f_{2}\right)\right\|_{L_{q}(E)} \\
& \lesssim\left\|b_{1}\right\|_{L C_{p_{1}, \lambda_{1}, P}^{\left\{x_{0}\right\}}}\left\|b_{2}\right\|_{L C_{p_{2}, \lambda_{2}, P}^{\left\{x_{0}\right\}}} r^{\frac{\gamma}{q}} \int_{2 k r}^{\infty}\left(1+\ln \frac{t}{r}\right)^{2} \frac{\|f\|_{L_{p}\left(E\left(x_{0}, t\right)\right)}}{t^{\frac{\gamma}{p}-\gamma\left(\lambda_{1}+\lambda_{2}\right)+1}} d t .
\end{aligned}
$$

So, combining all the estimates for $G_{1}, G_{2}, G_{3}, G_{4}$, we get

$$
G=\left\|\left[\left(b_{1}, b_{2}\right), T_{\Omega}^{P}\right] f_{2}\right\|_{L_{q}(E)} \lesssim\left\|b_{1}\right\|_{L C_{p_{1}, \lambda_{1}, P}^{\left\{x_{0}\right\}}}\left\|b_{2}\right\|_{L C_{p_{2}, \lambda_{2}, P}^{\left\{x_{0}\right\}}} r^{\frac{\gamma}{q}} \int_{2 k r}^{\infty}\left(1+\ln \frac{t}{r}\right)^{2} \frac{\|f\|_{L_{p}\left(E\left(x_{0}, t\right)\right)}}{t^{\frac{\gamma}{p}-\gamma\left(\lambda_{1}+\lambda_{2}\right)+1}} d t .
$$

Thus, putting estimates $F$ and $G$ together, we get the desired conclusion

$$
\left\|\left[\left(b_{1}, b_{2}\right), T_{\Omega}^{P}\right] f\right\|_{L_{q}(E)} \lesssim\left\|b_{1}\right\|_{L C_{p_{1}, \lambda_{1}, P}^{\left\{x_{0}\right\}}}\left\|b_{2}\right\|_{L C_{p_{2}, \lambda_{2}, P}^{\left\{x_{0}\right\}}} r^{\frac{\gamma}{q}} \int_{2 k r}^{\infty}\left(1+\ln \frac{t}{r}\right)^{2} \frac{\|f\|_{L_{p}\left(E\left(x_{0}, t\right)\right)}}{t^{\frac{\gamma}{p}-\gamma\left(\lambda_{1}+\lambda_{2}\right)+1}} d t .
$$

For the case of $p<s$, we can also use the same method, so we omit the details. This completes the proof of Lemma 2.2 .

Lemma 2.3. Suppose that $x_{0} \in \mathbb{R}^{n}, \Omega \in L_{s}\left(S^{n-1}\right), 1<s \leq \infty$, is $A_{t}$-homogeneous of degree zero. Let $T_{\Omega, \alpha}^{P}$ be a parabolic linear operator satisfying condition (1.2). Let also $0<\alpha<\gamma$ and $1<q, q_{1}, p_{i}, p<\frac{\gamma}{\alpha}$ with $\frac{1}{q}=\sum_{i=1}^{m} \frac{1}{p_{i}}+\frac{1}{p}, \frac{1}{q_{1}}=\frac{1}{q}-\frac{\alpha}{\gamma}$ and $\vec{b} \in L C_{p_{i}, \lambda_{i}, P}^{\left\{x_{0}\right\}}\left(\mathbb{R}^{n}\right)$ for $0 \leq \lambda_{i}<\frac{1}{\gamma}, i=1, \ldots, m$.

Then, for $s^{\prime} \leq q$ the inequality

$$
\left\|\left[\vec{b}, T_{\Omega, \alpha}^{P}\right] f\right\|_{L_{q_{1}}\left(E\left(x_{0}, r\right)\right)} \lesssim \prod_{i=1}^{m}\|\vec{b}\|_{L C_{p_{i}, \lambda_{i}, P}^{\left\{x_{0}\right\}}} r^{\frac{\gamma}{q_{1}}} \int_{2 k r}^{\infty}\left(1+\ln \frac{t}{r}\right)^{m} \frac{\|f\|_{L_{p}\left(E\left(x_{0}, t\right)\right)}}{\gamma\left(\frac{1}{q_{1}}-\left(\sum_{i=1}^{m} \lambda_{i}+\sum_{i=1}^{m} \frac{1}{p_{i}}\right)\right)+1} d t
$$

holds for any ellipsoid $E\left(x_{0}, r\right)$ and for all $f \in L_{p}^{\text {loc }}\left(\mathbb{R}^{n}\right)$. Also, for $q_{1}<s$ the inequality

$$
\begin{aligned}
\left\|\left[\vec{b}, T_{\Omega, \alpha}^{P}\right] f\right\|_{L_{q_{1}}\left(E\left(x_{0}, r\right)\right)} & \lesssim \prod_{i=1}^{m}\|\vec{b}\|_{L C_{p_{i}, \lambda_{i}, P}^{\left\{x_{0}\right\}}} r^{\frac{\gamma}{q_{1}}-\frac{\gamma}{s}} \int_{2 k r}^{\infty}\left(1+\ln \frac{t}{r}\right)^{m} \frac{\|f\|_{L_{p}\left(E\left(x_{0}, t\right)\right)}}{\gamma\left(\frac{1}{q_{1}}-\left(\frac{1}{s}+\sum_{i=1}^{m} \lambda_{i}+\sum_{i=1}^{m} \frac{1}{p_{i}}\right)\right)+1} d t
\end{aligned}
$$


holds for any ellipsoid $E\left(x_{0}, r\right)$ and for all $f \in L_{p}^{l o c}\left(\mathbb{R}^{n}\right)$.

Proof. Similar to the proof of Lemma 2.2, it is sufficient to show that the conclusion holds for $m=2$. As in the proof of Lemma 2.2, we split $f=f_{1}+f_{2}$ in form (2.5) and have

$$
\left\|\left[\left(b_{1}, b_{2}\right), T_{\Omega, \alpha}^{P}\right] f\right\|_{L_{q_{1}}(E)} \leq\left\|\left[\left(b_{1}, b_{2}\right), T_{\Omega, \alpha}^{P}\right] f_{1}\right\|_{L_{q_{1}}(E)}+\left\|\left[\left(b_{1}, b_{2}\right), T_{\Omega, \alpha}^{P}\right] f_{2}\right\|_{L_{q_{1}}(E)}=: A+B .
$$

Let us estimate $A+B$, respectively.

For $\left[\left(b_{1}, b_{2}\right), T_{\Omega, \alpha}^{P}\right] f_{1}(x)$, it is easy to see that

$$
\begin{aligned}
A=\left\|\left[\left(b_{1}, b_{2}\right), T_{\Omega, \alpha}^{P}\right] f_{1}\right\|_{L_{q_{1}}(E)} \lesssim & \left\|\left(b_{1}-\left(b_{1}\right)_{E}\right)\left(b_{2}(x)-\left(b_{2}\right)_{E}\right) T_{\Omega, \alpha}^{P} f_{1}\right\|_{L_{q_{1}}(E)} \\
& +\left\|\left(b_{1}-\left(b_{1}\right)_{E}\right) T_{\Omega, \alpha}^{P}\left(\left(b_{2}-\left(b_{2}\right)_{E}\right) f_{1}\right)\right\|_{L_{q_{1}}(E)} \\
& +\left\|\left(b_{2}-\left(b_{2}\right)_{E}\right) T_{\Omega, \alpha}^{P}\left(\left(b_{1}-\left(b_{1}\right)_{E}\right) f_{1}\right)\right\|_{L_{q_{1}}(E)} \\
& +\left\|T_{\Omega, \alpha}^{P}\left(\left(b_{1}-\left(b_{1}\right)_{E}\right)\left(b_{2}-\left(b_{2}\right)_{E}\right) f_{1}\right)\right\|_{L_{q_{1}}(E)} \\
\equiv & A_{1}+A_{2}+A_{3}+A_{4} .
\end{aligned}
$$

Let $1<\bar{q}<\infty$. Since $\frac{1}{\bar{q}}=\frac{1}{p}-\frac{\alpha}{\gamma}$, it is obvious that $\frac{1}{q_{1}}=\frac{1}{p_{1}}+\frac{1}{p_{2}}+\frac{1}{\bar{q}}$. Thus, using Hölder's inequality and by Theorem 0.1 in [8] and (2.7) it follows that:

$$
\begin{aligned}
A_{1} & \lesssim\left\|\left(b_{1}-\left(b_{1}\right)_{E}\right)\left(b_{2}-\left(b_{2}\right)_{E}\right)\right\|_{L_{\bar{r}}(E)}\left\|T_{\Omega, \alpha}^{P} f_{1}\right\|_{L_{\bar{q}}(E)} \\
& \lesssim\left\|b_{1}-\left(b_{1}\right)_{B}\right\|_{L_{p_{1}}(E)}\left\|b_{2}-\left(b_{2}\right)_{B}\right\|_{L_{p_{2}}(E)}\|f\|_{L_{p}(2 k E)} \\
& \lesssim\left\|b_{1}-\left(b_{1}\right)_{E}\right\|_{L_{p_{1}}(E)}\left\|b_{2}-\left(b_{2}\right)_{E}\right\|_{L_{p_{2}}(E)} r^{\frac{\gamma}{\bar{q}}} \int_{2 k r}^{\infty}\|f\|_{L_{p}\left(E\left(x_{0}, t\right)\right)} \frac{d t}{t^{\frac{\gamma}{\bar{q}}+1}} \\
& \lesssim\left\|b_{1}\right\|_{L C_{p_{1}, \lambda_{1}, P}^{\left\{x_{0}\right\}}}\left\|b_{2}\right\|_{L C_{p_{2}, \lambda_{2}, P}^{\left\{x_{0}\right\}}} r^{\frac{\gamma}{q_{1}}} \int_{2 k r}^{\infty}\left(1+\ln \frac{t}{r}\right)^{2} \frac{\|f\|_{L_{p}\left(E\left(x_{0}, t\right)\right)}}{t^{\frac{\gamma}{q_{1}}-\gamma\left(\lambda_{1}+\lambda_{2}\right)-\gamma\left(\frac{1}{p_{1}}+\frac{1}{p_{2}}\right)+1}} d t .
\end{aligned}
$$

To estimate $A_{2}$, let $1<\tau<\infty$, such that $\frac{1}{q_{1}}=\frac{1}{p_{1}}+\frac{1}{\tau}$. Then, similar to the estimates for $A_{1}$, we have

$$
\begin{aligned}
A_{2} & \lesssim\left\|b_{1}-\left(b_{1}\right)_{E}\right\|_{L_{p_{1}}(E)}\left\|T_{\Omega, \alpha}^{P}\left(\left(b_{2}(\cdot)-\left(b_{2}\right)_{E}\right) f_{1}\right)\right\|_{L_{\tau}(E)} \\
& \lesssim\left\|b_{1}-\left(b_{1}\right)_{E}\right\|_{L_{p_{1}}(E)}\left\|\left(b_{2}(\cdot)-\left(b_{2}\right)_{E}\right) f_{1}\right\|_{L_{k}(E)} \\
& \lesssim\left\|b_{1}-\left(b_{1}\right)_{E}\right\|_{L_{p_{1}}(E)}\left\|b_{2}-\left(b_{2}\right)_{E}\right\|_{L_{p_{2}}(2 k E)}\|f\|_{L_{p}(2 k E)},
\end{aligned}
$$

where $1<k<\frac{2 \gamma}{\alpha}$, such that $\frac{1}{k}=\frac{1}{p_{2}}+\frac{1}{p}=\frac{1}{\tau}+\frac{\alpha}{\gamma}$. By (2.7) and (2.8), we get

$$
A_{2} \lesssim\left\|b_{1}\right\|_{L C_{p_{1}, \lambda_{1}, P}^{\left\{x_{0}\right\}}}\left\|b_{2}\right\|_{L C_{p_{2}, \lambda_{2}, P}^{\left\{x_{0}\right\}}} r^{\frac{\gamma}{q_{1}}} \int_{2 k r}^{\infty}\left(1+\ln \frac{t}{r}\right)^{2} \frac{\|f\|_{L_{p}\left(E\left(x_{0}, t\right)\right)}}{t^{\frac{\gamma}{q_{1}}-\gamma\left(\lambda_{1}+\lambda_{2}\right)-\gamma\left(\frac{1}{p_{1}}+\frac{1}{p_{2}}\right)+1}} d t .
$$

In a similar way, $A_{3}$ has the same estimate as above, so we omit the details. Then we have that

$$
A_{3} \lesssim\left\|b_{1}\right\|_{L C_{p_{1}, \lambda_{1}, P}^{\left\{x_{0}\right\}}}\left\|b_{2}\right\|_{L C_{p_{2}, \lambda_{2}, P}^{\left\{x_{0}\right\}}} r^{\frac{\gamma}{q_{1}}} \int_{2 k r}^{\infty}\left(1+\ln \frac{t}{r}\right)^{2} \frac{\|f\|_{L_{p}\left(E\left(x_{0}, t\right)\right)}}{t^{\frac{\gamma}{q_{1}}-\gamma\left(\lambda_{1}+\lambda_{2}\right)-\gamma\left(\frac{1}{p_{1}}+\frac{1}{p_{2}}\right)+1}} d t .
$$

Now let us consider the term $A_{4}$. Let $1<q<\frac{2 \gamma}{\alpha}$ such that $\frac{1}{q_{1}}=\frac{1}{q}-\frac{\alpha}{\gamma}$. It is easy to see that $\frac{1}{q}=\frac{1}{p_{1}}+\frac{1}{p_{2}}+\frac{1}{p}$. Thus, by Theorem 0.1 in [8], Hölder's inequality and (2.8), we 
obtain

$$
\begin{aligned}
A_{4} & \lesssim\left\|\left(b_{1}-\left(b_{1}\right)_{E}\right)\left(b_{2}-\left(b_{2}\right)_{E}\right) f_{1}\right\|_{L_{q}(E)} \\
& \lesssim\left\|b_{1}-\left(b_{1}\right)_{E}\right\|_{L_{p_{1}}(2 k E)}\left\|b_{2}-\left(b_{2}\right)_{E}\right\|_{L_{p_{2}}(2 k E)}\|f\|_{L_{p}(2 k E)} \\
& \lesssim\left\|b_{1}\right\|_{L C_{p_{1}, \lambda_{1}, P}^{\left\{x_{0}\right\}}}\left\|b_{2}\right\|_{L C_{p_{2}, \lambda_{2}, P}^{\left\{x_{0}\right\}}} r^{\frac{\gamma}{q_{1}}} \int_{2 k r}^{\infty}\left(1+\ln \frac{t}{r}\right)^{2} \frac{\|f\|_{L_{p}\left(E\left(x_{0}, t\right)\right)}}{t^{\frac{\gamma}{q_{1}}-\gamma\left(\lambda_{1}+\lambda_{2}\right)-\gamma\left(\frac{1}{p_{1}}+\frac{1}{p_{2}}\right)+1}} d t .
\end{aligned}
$$

Combining all the estimates of $A_{1}, A_{2}, A_{3}, A_{4}$; we get

$$
\begin{aligned}
A= & \left\|\left[\left(b_{1}, b_{2}\right), T_{\Omega, \alpha}^{P}\right] f_{1}\right\|_{L_{q_{1}}(E)} \\
& \lesssim\left\|b_{1}\right\|_{L C_{p_{1}, \lambda_{1}, P}^{\left\{x_{0}\right\}}}\left\|b_{2}\right\|_{L C_{p_{2}, \lambda_{2}, P}^{\left\{x_{0}\right\}}} r^{\frac{\gamma}{q_{1}}} \int_{2 k r}^{\infty}\left(1+\ln \frac{t}{r}\right)^{2} \frac{\|f\|_{L_{p}\left(E\left(x_{0}, t\right)\right)}}{t^{\frac{\gamma}{q_{1}}-\gamma\left(\lambda_{1}+\lambda_{2}\right)-\gamma\left(\frac{1}{p_{1}}+\frac{1}{p_{2}}\right)+1}} d t .
\end{aligned}
$$

Now, let us estimate $B=\left\|\left[\left(b_{1}, b_{2}\right), T_{\Omega, \alpha}^{P}\right] f_{2}\right\|_{L_{q_{1}}(E)}$. For $B$, it's similar to (2.11) we also write

$$
\begin{aligned}
B=\left\|\left[\left(b_{1}, b_{2}\right), T_{\Omega, \alpha}^{P}\right] f_{2}\right\|_{L_{q_{1}}(E) \lesssim} & \left\|\left(b_{1}-\left(b_{1}\right)_{E}\right)\left(b_{2}(x)-\left(b_{2}\right)_{E}\right) T_{\Omega, \alpha}^{P} f_{2}\right\|_{L_{q_{1}}(E)} \\
& +\left\|\left(b_{1}-\left(b_{1}\right)_{E}\right) T_{\Omega, \alpha}^{P}\left(\left(b_{2}-\left(b_{2}\right)_{E}\right) f_{2}\right)\right\|_{L_{q_{1}}(E)} \\
& +\left\|\left(b_{2}-\left(b_{2}\right)_{E}\right) T_{\Omega, \alpha}^{P}\left(\left(b_{1}-\left(b_{1}\right)_{E}\right) f_{2}\right)\right\|_{L_{q_{1}}(E)} \\
& +\left\|T_{\Omega, \alpha}^{P}\left(\left(b_{1}-\left(b_{1}\right)_{E}\right)\left(b_{2}-\left(b_{2}\right)_{E}\right) f_{2}\right)\right\|_{L_{q_{1}}(E)} \\
\equiv & B_{1}+B_{2}+B_{3}+B_{4} .
\end{aligned}
$$

Let $1<p_{1}, p_{2}<\frac{2 \gamma}{\alpha}$. Since $\frac{1}{\bar{q}}=\frac{1}{p}-\frac{\alpha}{\gamma}$, it is easy to see that $\frac{1}{q_{1}}=\frac{1}{p_{1}}+\frac{1}{p_{2}}+\frac{1}{\bar{q}}$. Thus, using Hölder's inequality and by $(2.6)$ in [8] and (2.7), we have

$$
\begin{aligned}
B_{1} & \lesssim\left\|\left(b_{1}-\left(b_{1}\right)_{E}\right)\left(b_{2}-\left(b_{2}\right)_{E}\right)\right\|_{L_{\bar{p}}(E)}\left\|T_{\Omega, \alpha}^{P} f_{2}\right\|_{L_{\bar{q}}(E)} \\
& \lesssim\left\|b_{1}-\left(b_{1}\right)_{E}\right\|_{L_{p_{1}}(E)}\left\|b_{2}-\left(b_{2}\right)_{E}\right\|_{L_{p_{2}}(E)} r^{\frac{\gamma}{\bar{q}}} \int_{2 k r}^{\infty}\|f\|_{L_{p}\left(E\left(x_{0}, t\right)\right)} t^{-\frac{\gamma}{\bar{q}}-1} d t \\
& \lesssim\left\|b_{1}\right\|_{L C_{p_{1}, \lambda_{1}, P}^{\left\{x_{0}\right\}}}\left\|b_{2}\right\|_{L C_{p_{2}, \lambda_{2}, P}^{\left\{x_{0}\right\}}} r^{\frac{\gamma}{q_{1}}} \int_{2 k r}^{\infty}\left(1+\ln \frac{t}{r}\right)^{2} \frac{\|f\|_{L_{p}\left(E\left(x_{0}, t\right)\right)}}{t^{\frac{\gamma}{q_{1}}-\gamma\left(\lambda_{1}+\lambda_{2}\right)-\gamma\left(\frac{1}{p_{1}}+\frac{1}{p_{2}}\right)+1}} d t .
\end{aligned}
$$

On the other hand, for the estimates used in $B_{2}, B_{3}$, we have to prove the below inequality:

$$
\left|T_{\Omega, \alpha}^{P}\left(\left(b_{2}-\left(b_{2}\right)_{E}\right) f_{2}\right)(x)\right| \lesssim\left\|b_{2}\right\|_{L C_{p_{2}, \lambda_{2}, P}^{\left\{x_{0}\right\}}} \int_{2 k r}^{\infty}\left(1+\ln \frac{t}{r}\right) t^{-\frac{\gamma}{p}+\gamma \lambda_{2}-1+\alpha}\|f\|_{L_{p}\left(E\left(x_{0}, t\right)\right)} d t .
$$

Indeed, when $s^{\prime} \leq q$, for $x \in E$, by Fubini's theorem and applying Hölder's inequality and from (2.2), (2.3), $0<r<t$ and (11) in [7] we have

$$
\begin{aligned}
& \left|T_{\Omega, \alpha}^{P}\left(\left(b_{2}(\cdot)-\left(b_{2}\right)_{E}\right) f_{2}\right)(x)\right| \\
& \lesssim \int_{(2 k E)^{C}}\left|b_{2}(y)-\left(b_{2}\right)_{E}\right||\Omega(x-y)| \frac{|f(y)|}{\rho\left(x_{0}-y\right)^{\gamma-\alpha}} d y
\end{aligned}
$$




$$
\begin{aligned}
& \approx \int_{2 k r 2 k r<\rho\left(x_{0}-y\right)<t}^{\infty}\left|b_{2}(y)-\left(b_{2}\right)_{E}\right||\Omega(x-y)||f(y)| d y \frac{d t}{t^{\gamma-\alpha+1}} \\
& \lesssim \int_{2 k r E\left(x_{0}, t\right)}^{\infty}\left|b_{2}(y)-\left(b_{2}\right)_{E\left(x_{0}, t\right)}\right||\Omega(x-y)||f(y)| d y \frac{d t}{t^{\gamma-\alpha+1}} \\
& +\int_{2 k r}^{\infty}\left|\left(b_{2}\right)_{E\left(x_{0}, t\right)}-\left(b_{2}\right)_{E\left(x_{0}, r\right) \mid} \int_{E\left(x_{0}, t\right)}\right| \Omega(x-y)|| f(y) \mid d y \frac{d t}{t^{\gamma-\alpha+1}} \\
& \lesssim \int_{2 k r}^{\infty}\left\|b_{2}(\cdot)-\left(b_{2}\right)_{E\left(x_{0}, t\right)}\right\|_{L_{p_{2}}\left(E\left(x_{0}, t\right)\right)}\|\Omega(\cdot-y)\|_{L_{s}\left(E\left(x_{0}, t\right)\right)}\|f\|_{L_{p}\left(E\left(x_{0}, t\right)\right)} \\
& +\int_{2 k r}^{\infty}\left|\left(b_{2}\right)_{E\left(x_{0}, t\right)}-\left(b_{2}\right)_{E\left(x_{0}, r\right)}\right|\|f\|_{L_{p}\left(E\left(x_{0}, t\right)\right)}\|\Omega(\cdot-y)\|_{L_{s}\left(E\left(x_{0}, t\right)\right)}\left|E\left(x_{0}, t\right)\right|^{1-\frac{1}{p}-\frac{1}{s}} \frac{d t}{t^{\gamma-\alpha+1}} \\
& \lesssim\left\|b_{2}\right\|_{L C_{p_{2}, \lambda_{2}, P}^{\left\{x_{2}\right\}}} \int_{2 k r}^{\infty}\left(1+\ln \frac{t}{r}\right) t^{-\frac{\gamma}{p}+\gamma \lambda_{2}-1+\alpha}\|f\|_{L_{p}\left(E\left(x_{0}, t\right)\right)} d t .
\end{aligned}
$$

This completes the proof of inequality (2.12).

Let $1<\tau<\infty$, such that $\frac{1}{q_{1}}=\frac{1}{p_{1}}+\frac{1}{\tau}$ and $\frac{1}{\tau}=\frac{1}{p_{2}}+\frac{1}{p}-\frac{\alpha}{\gamma}$. Then, using Hölder's inequality and from (2.12), (2.3) and $0<r<t$, we get

$$
\begin{aligned}
B_{2} & \lesssim\left\|b_{1}-\left(b_{1}\right)_{E}\right\|_{L_{p_{1}}(E)}\left\|T_{\Omega, \alpha}^{P}\left(\left(b_{2}(\cdot)-\left(b_{2}\right)_{E}\right) f_{2}\right)\right\|_{L_{\tau}(E)} \\
& \lesssim\left\|b_{1}\right\|_{L C_{p_{1}, \lambda_{1}, P}^{\left\{x_{0}\right\}}}\left\|b_{2}\right\|_{L C_{p_{2}, \lambda_{2}, P}^{\left\{x_{0}\right\}}} r^{\frac{\gamma}{q_{1}}} \int_{2 k r}^{\infty}\left(1+\ln \frac{t}{r}\right)^{2} \frac{\|f\|_{L_{p}\left(E\left(x_{0}, t\right)\right)}}{t^{\frac{\gamma}{q_{1}}-\gamma\left(\lambda_{1}+\lambda_{2}\right)-\gamma\left(\frac{1}{p_{1}}+\frac{1}{p_{2}}\right)+1}} d t .
\end{aligned}
$$

Similarly, $B_{3}$ has the same estimate above, so here we omit the details. Then the inequality

$$
\begin{aligned}
B_{3} & =\left\|\left(b_{2}-\left(b_{2}\right)_{E}\right) T_{\Omega, \alpha}^{P}\left(\left(b_{1}-\left(b_{1}\right)_{E}\right) f_{2}\right)\right\|_{L_{q_{1}}(E)} \\
& \lesssim\left\|b_{1}\right\|_{L C_{p_{1}, \lambda_{1}, P}^{\left\{x_{0}\right\}}}\left\|b_{2}\right\|_{L C_{p_{2}, \lambda_{2}, P}^{\left\{x_{0}\right\}}} r^{\frac{\gamma}{q_{1}}} \int_{2 k r}^{\infty}\left(1+\ln \frac{t}{r}\right)^{2} \frac{\|f\|_{L_{p}\left(E\left(x_{0}, t\right)\right)}}{t^{\frac{\gamma}{q_{1}}-\gamma\left(\lambda_{1}+\lambda_{2}\right)-\gamma\left(\frac{1}{p_{1}}+\frac{1}{p_{2}}\right)+1}} d t
\end{aligned}
$$

is valid.

Now, let us estimate $B_{4}=\left\|T_{\Omega, \alpha}^{P}\left(\left(b_{1}-\left(b_{1}\right)_{E}\right)\left(b_{2}-\left(b_{2}\right)_{E}\right) f_{2}\right)\right\|_{L_{q_{1}}(E)}$. It's similar to the estimate of (2.12), for any $x \in E$, we also write

$$
\begin{aligned}
& \left|T_{\Omega, \alpha}^{P}\left(\left(b_{1}-\left(b_{1}\right)_{E}\right)\left(b_{2}-\left(b_{2}\right)_{E}\right) f_{2}\right)(x)\right| \\
& \lesssim \int_{2 k r E\left(x_{0}, t\right)}^{\infty}\left|b_{1}(y)-\left(b_{1}\right)_{E\left(x_{0}, t\right)}\right|\left|b_{2}(y)-\left(b_{2}\right)_{E\left(x_{0}, t\right)}\right||\Omega(x-y)||f(y)| d y \frac{d t}{t^{\gamma-\alpha+1}} \\
& +\int_{2 k r E\left(x_{0}, t\right)}^{\infty}\left|b_{1}(y)-\left(b_{1}\right)_{E\left(x_{0}, t\right)}\right|\left|\left(b_{2}\right)_{E\left(x_{0}, t\right)}-\left(b_{2}\right)_{E\left(x_{0}, r\right)}\right||\Omega(x-y)||f(y)| d y \frac{d t}{t^{\gamma-\alpha+1}} \\
& +\int_{2 k r E\left(x_{0}, t\right)}^{\infty}\left|\left(b_{1}\right)_{E\left(x_{0}, t\right)}-\left(b_{2}\right)_{E\left(x_{0}, r\right)}\right|\left|b_{2}(y)-\left(b_{2}\right)_{E\left(x_{0}, t\right)}\right||\Omega(x-y)||f(y)| d y \frac{d t}{t^{\gamma-\alpha+1}} \\
& +\int_{2 k r E\left(x_{0}, t\right)}^{\infty}\left|\left(b_{1}\right)_{E\left(x_{0}, t\right)}-\left(b_{2}\right)_{E\left(x_{0}, r\right)}\right|\left|\left(b_{2}\right)_{E\left(x_{0}, t\right)}-\left(b_{2}\right)_{E\left(x_{0}, r\right)}\right||\Omega(x-y)||f(y)| d y \frac{d t}{t^{\gamma-\alpha+1}}
\end{aligned}
$$


$\equiv B_{41}+B_{42}+B_{43}+B_{44}$.

Let us estimate $B_{41}, B_{42}, B_{43}, B_{44}$, respectively.

Firstly, to estimate $B_{41}$, similar to the estimate of (2.12), we get

$$
B_{41} \lesssim\left\|b_{1}\right\|_{L C_{p_{1}, \lambda_{1}, P}^{\left\{x_{0}\right\}}}\left\|b_{2}\right\|_{L C_{p_{2}, \lambda_{2}, P}^{\left\{x_{0}\right\}}} \int_{2 k r}^{\infty}\left(1+\ln \frac{t}{r}\right)^{2} \frac{\|f\|_{L_{p}\left(E\left(x_{0}, t\right)\right)}}{t^{\frac{\gamma}{q_{1}}-\gamma\left(\lambda_{1}+\lambda_{2}\right)-\gamma\left(\frac{1}{p_{1}}+\frac{1}{p_{2}}\right)+1}} d t .
$$

Secondly, to estimate $B_{42}$ and $B_{43}$, from (2.12), (2.2), (2.3) and $0<r<t$, it follows that

$$
B_{42} \lesssim\left\|b_{1}\right\|_{L C_{p_{1}, \lambda_{1}, P}^{\left\{x_{0}\right\}}}\left\|b_{2}\right\|_{L C_{p_{2}, \lambda_{2}, P}^{\left\{x_{0}\right\}}} \int_{2 k r}^{\infty}\left(1+\ln \frac{t}{r}\right)^{2} \frac{\|f\|_{L_{p}\left(E\left(x_{0}, t\right)\right)}}{t^{\frac{\gamma}{q_{1}}-\gamma\left(\lambda_{1}+\lambda_{2}\right)-\gamma\left(\frac{1}{p_{1}}+\frac{1}{p_{2}}\right)+1}} d t,
$$

and

$$
B_{43} \lesssim\left\|b_{1}\right\|_{L C_{p_{1}, \lambda_{1}, P}^{\left\{x_{0}\right\}}}\left\|b_{2}\right\|_{L C_{p_{2}, \lambda_{2}, P}^{\left\{x_{0}\right\}}} \int_{2 k r}^{\infty}\left(1+\ln \frac{t}{r}\right)^{2} \frac{\|f\|_{L_{p}\left(E\left(x_{0}, t\right)\right)}}{t^{\frac{\gamma}{q_{1}}-\gamma\left(\lambda_{1}+\lambda_{2}\right)-\gamma\left(\frac{1}{p_{1}}+\frac{1}{p_{2}}\right)+1}} d t .
$$

Finally, to estimate $B_{44}$, similar to the estimate of (2.12) and from (2.2), (2.3) and $0<r<t$, we have

$$
B_{44} \lesssim\left\|b_{1}\right\|_{L C_{p_{1}, \lambda_{1}, P}^{\left\{x_{0}\right\}}}\left\|b_{2}\right\|_{L C_{p_{2}, \lambda_{2}, P}^{\left\{x_{0}\right\}}} \int_{2 k r}^{\infty}\left(1+\ln \frac{t}{r}\right)^{2} \frac{\|f\|_{L_{p}\left(E\left(x_{0}, t\right)\right)}}{t^{\frac{\gamma}{q_{1}}-\gamma\left(\lambda_{1}+\lambda_{2}\right)-\gamma\left(\frac{1}{p_{1}}+\frac{1}{p_{2}}\right)+1}} d t .
$$

By the estimates of $B_{4 j}$ above, where $j=1,2,3$, we know that

$$
\begin{aligned}
\left|T_{\Omega, \alpha}^{P}\left(\left(b_{1}-\left(b_{1}\right)_{E}\right)\left(b_{2}-\left(b_{2}\right)_{E}\right) f_{2}\right)(x)\right| & \lesssim\left\|b_{1}\right\|_{L C_{p_{1}, \lambda_{1}, P}^{\left\{x_{0}\right\}}\left\|b_{2}\right\|_{L C_{p_{2}, \lambda_{2}, P}^{\left\{x_{0}\right\}}} \int_{2 k r}^{\infty}\left(1+\ln \frac{t}{r}\right)^{2}} \\
& \times \frac{\|f\|_{L_{p}\left(E\left(x_{0}, t\right)\right)}}{t^{\frac{\gamma}{q_{1}}-\gamma\left(\lambda_{1}+\lambda_{2}\right)-\gamma\left(\frac{1}{p_{1}}+\frac{1}{p_{2}}\right)+1}} d t .
\end{aligned}
$$

Then, we have

$$
B_{4} \lesssim\left\|b_{1}\right\|_{L C_{p_{1}, \lambda_{1}, P}^{\left\{x_{0}\right\}}}\left\|b_{2}\right\|_{L C_{p_{2}, \lambda_{2}, P}^{\left\{x_{0}\right\}}} r^{\frac{\gamma}{q_{1}}} \int_{2 k r}^{\infty}\left(1+\ln \frac{t}{r}\right)^{2} \frac{\|f\|_{L_{p}\left(E\left(x_{0}, t\right)\right)}}{t^{\frac{\gamma}{q_{1}}-\gamma\left(\lambda_{1}+\lambda_{2}\right)-\gamma\left(\frac{1}{p_{1}}+\frac{1}{p_{2}}\right)+1}} d t .
$$

So, combining all the estimates for $B_{1}, B_{2}, B_{3}, B_{4}$, we get

$$
\begin{aligned}
B= & \left\|\left[\left(b_{1}, b_{2}\right), T_{\Omega, \alpha}^{P}\right] f_{2}\right\|_{L_{q_{1}}(E)} \\
& \lesssim\left\|b_{1}\right\|_{L C_{p_{1}, \lambda_{1}, P}^{\left\{x_{0}\right\}}}\left\|b_{2}\right\|_{L C_{p_{2}, \lambda_{2}, P}^{\left\{x_{0}\right\}}} r^{\frac{\gamma}{q_{1}}} \int_{2 k r}^{\infty}\left(1+\ln \frac{t}{r}\right)^{2} \frac{\|f\|_{L_{p}\left(E\left(x_{0}, t\right)\right)}}{t^{\frac{\gamma}{q_{1}}-\gamma\left(\lambda_{1}+\lambda_{2}\right)-\gamma\left(\frac{1}{p_{1}}+\frac{1}{p_{2}}\right)+1}} d t .
\end{aligned}
$$

Thus, putting estimates $A$ and $B$ together, we get the desired conclusion

$$
\begin{aligned}
& \left\|\left[\left(b_{1}, b_{2}\right), T_{\Omega, \alpha}^{P}\right] f\right\|_{L_{q_{1}}(E)} \\
& \quad \lesssim\left\|b_{1}\right\|_{L C_{p_{1}, \lambda_{1}, P}^{\left\{x_{0}\right\}}}\left\|b_{2}\right\|_{L C_{p_{2}, \lambda_{2}, P}^{\left\{x_{0}\right\}}} r^{\frac{\gamma}{q_{1}}} \int_{2 k r}^{\infty}\left(1+\ln \frac{t}{r}\right)^{2} \frac{\|f\|_{L_{p}\left(E\left(x_{0}, t\right)\right)}}{t^{\frac{\gamma}{q_{1}}-\gamma\left(\lambda_{1}+\lambda_{2}\right)-\gamma\left(\frac{1}{p_{1}}+\frac{1}{p_{2}}\right)+1}} d t .
\end{aligned}
$$

For the case of $q_{1}<s$, we can also use the same method, so we omit the details. Thus, we complete the the proof of Lemma 2.3. 


\section{Proofs of the main results}

\subsection{Proof of Theorem 1.3.}

We consider (1.5) firstly. Since $f \in L M_{p, \varphi_{1}, P}^{\left\{x_{0}\right\}}$, by (1.3) and it is also non-decreasing, with respect to $t$, of the norm $\|f\|_{L_{p}\left(E\left(x_{0}, t\right)\right)}$, we get

$$
\begin{aligned}
\frac{\|f\|_{L_{p}\left(E\left(x_{0}, t\right)\right)}}{\underset{0<t<\tau<\infty}{\operatorname{essinf}} \varphi_{1}\left(x_{0}, \tau\right) \tau^{\frac{\gamma}{p}}} & \leq \operatorname{esssup}_{0<t<\tau<\infty} \frac{\|f\|_{L_{p}\left(E\left(x_{0}, t\right)\right)}}{\varphi_{1}\left(x_{0}, \tau\right) \tau^{\frac{\gamma}{p}}} \\
& \leq \operatorname{esssup}_{0<\tau<\infty} \frac{\|f\|_{L_{p}\left(E\left(x_{0}, \tau\right)\right)}}{\varphi_{1}\left(x_{0}, \tau\right) \tau^{\frac{\gamma}{p}}} \leq\|f\|_{L M_{p, \varphi, P}^{\left\{x_{0}\right\}}} .
\end{aligned}
$$

For $s^{\prime} \leq q<\infty$, since $\left(\varphi_{1}, \varphi_{2}\right)$ satisfies (1.4), we have

$$
\begin{aligned}
& \int_{r}^{\infty}\left(1+\ln \frac{t}{r}\right)^{m} \frac{\|f\|_{L_{p}\left(E\left(x_{0}, t\right)\right)}}{\gamma\left(\frac{1}{p}-\sum_{i=1}^{m} \lambda_{i}\right)+1} d t \\
& \leq \int_{r}^{\infty}\left(1+\ln \frac{t}{r}\right)^{m} \frac{\|f\|_{L_{p}\left(E\left(x_{0}, t\right)\right)}}{\operatorname{essinf}_{t<\tau<\infty} \varphi_{1}\left(x_{0}, \tau\right) \tau^{\frac{\gamma}{p}}} \frac{\operatorname{essinf}_{t<\tau<\infty} \varphi_{1}\left(x_{0}, \tau\right) \tau^{\frac{\gamma}{p}}}{\gamma\left(\frac{1}{p}-\sum_{i=1}^{m} \lambda_{i}\right)+1} d t \\
& \leq C\|f\|_{L M_{p, \varphi, P}^{\left\{x_{0}\right\}}} \int_{r}^{\infty}\left(1+\ln \frac{t}{r}\right)^{m} \frac{\operatorname{essinf}_{t<\tau<\infty} \varphi_{1}\left(x_{0}, \tau\right) \tau^{\frac{\gamma}{p}}}{\gamma\left(\frac{1}{p}-\sum_{i=1}^{m} \lambda_{i}\right)+1} d t \\
& \leq C\|f\|_{L M_{p, \varphi, P}^{\left\{x_{0}\right\}}} \varphi_{2}\left(x_{0}, r\right) .
\end{aligned}
$$

Then by (2.4) and (3.2), we get

$$
\begin{aligned}
\left\|\left[\vec{b}, T_{\Omega}^{P}\right] f\right\|_{L M_{q, \varphi_{2}, P}^{\left\{x_{0}\right\}}}= & \sup _{r>0} \varphi_{2}\left(x_{0}, r\right)^{-1}\left|E\left(x_{0}, r\right)\right|^{-\frac{1}{q}}\left\|\left[\vec{b}, T_{\Omega}^{P}\right] f\right\|_{L_{q}\left(E\left(x_{0}, r\right)\right)} \\
\leq & C \prod_{i=1}^{m}\|\vec{b}\|_{L C_{p_{i}, \lambda_{i}, P}^{\left\{x_{0}\right\}}} \sup _{r>0} \varphi_{2}\left(x_{0}, r\right)^{-1} \\
& \times \int_{r}^{\infty}\left(1+\ln \frac{t}{r}\right)^{m} \frac{\|f\|_{L_{p}\left(E\left(x_{0}, t\right)\right)}}{\gamma\left(\frac{1}{p}-\sum_{i=1}^{m} \lambda_{i}\right)+1} d t \\
\leq & C \prod_{i=1}^{m}\|\vec{b}\|_{L C_{p_{i}, \lambda_{i}, P}^{\left\{x_{0}\right\}}}\|f\|_{L M_{p, \varphi, P}^{\left\{x_{0}\right\}} .}
\end{aligned}
$$

For the case of $p<s$, we can also use the same method, so we omit the details. Thus, we finish the proof of (1.5).

We are now in a place of proving (1.6) in Theorem 1.3.

Remark 3.1. The conclusion of (1.6) is a direct consequence of the following Lemma 3.2 and (1.5). In order to do this, we need to define an operator by

$$
\left[\vec{b}, \tilde{T}_{|\Omega|}^{P}\right](|f|)(x)=\int_{\mathbb{R}^{n}} \prod_{i=1}^{m}\left[\left|b_{i}(x)-b_{i}(y)\right|\right] \frac{|\Omega(x-y)|}{\rho(x-y)^{\gamma}}|f(y)| d y,
$$

where $\Omega \in L_{s}\left(S^{n-1}\right), 1<s \leq \infty$, is $A_{t}$-homogeneous of degree zero in $\mathbb{R}^{n}$. 
Using the idea of proving Lemma 2 in [4] (see also [9]), we can obtain the following pointwise relation:

Lemma 3.2. Let $\Omega \in L_{s}\left(S^{n-1}\right), 1<s \leq \infty$, be $A_{t}$-homogeneous of degree zero. Then we have

$$
M_{\Omega, \vec{b}}^{P} f(x) \leq\left[\vec{b}, \tilde{T}_{|\Omega|}^{P}\right](|f|)(x) \quad \text { for } x \in \mathbb{R}^{n} .
$$

In fact, for any $t>0$, we have

$$
\begin{aligned}
{\left[\vec{b}, \tilde{T}_{|\Omega|}^{P}\right](|f|)(x) } & \geq \int_{\rho(x-y)<t} \prod_{i=1}^{m}\left[\left|b_{i}(x)-b_{i}(y)\right|\right] \frac{|\Omega(x-y)|}{\rho(x-y)^{\gamma}}|f(y)| d y \\
& \geq \frac{1}{t^{\gamma}} \int_{E(x, t)} \prod_{i=1}^{m}\left[\left|b_{i}(x)-b_{i}(y)\right|\right]|\Omega(x-y)||f(y)| d y .
\end{aligned}
$$

Taking the supremum for $t>0$ on the inequality above, we get

$$
\left[\vec{b}, \tilde{T}_{|\Omega|}^{P}\right](|f|)(x) \geq M_{\Omega, \vec{b}}^{P} f(x) \quad \text { for } x \in \mathbb{R}^{n} .
$$

From the process proving (1.5), it is easy to see that the conclusions of (1.5) also hold for $\left[\vec{b}, \tilde{T}_{|\Omega|}^{P}\right]$. Combining this with Lemma 3.2 , we can immediately obtain (1.6), which completes the proof.

\subsection{Proof of Theorem 1.5.}

Similar to the proof of Theorem 1.3, We consider (1.8) firstly.

For $s^{\prime} \leq q<\infty$, since $\left(\varphi_{1}, \varphi_{2}\right)$ satisfies (1.7) and by (3.1), we have

$$
\begin{aligned}
& \int_{r}^{\infty}\left(1+\ln \frac{t}{r}\right)^{m} \frac{\|f\|_{L_{p}\left(E\left(x_{0}, t\right)\right)}}{\gamma\left(\frac{1}{q_{1}}-\left(\sum_{i=1}^{m} \lambda_{i}+\sum_{i=1}^{m} \frac{1}{p_{i}}\right)\right)+1} d t \\
& \leq \int_{r}^{\infty}\left(1+\ln \frac{t}{r}\right)^{m} \frac{\|f\|_{L_{p}\left(E\left(x_{0}, t\right)\right)}}{\operatorname{essinf}_{t<\tau<\infty} \varphi_{1}\left(x_{0}, \tau\right) \tau^{\frac{\gamma}{p}}} \frac{\operatorname{essinf}_{t<\tau<\infty} \varphi_{1}\left(x_{0}, \tau\right) \tau^{\frac{\gamma}{p}}}{\gamma\left(\frac{1}{q_{1}}-\left(\sum_{i=1}^{m} \lambda_{i}+\sum_{i=1}^{m} \frac{1}{p_{i}}\right)\right)+1} d t \\
& \leq C\|f\|_{L M_{p, \varphi, P}^{\left\{x_{0}\right\}} \int_{r}^{\infty}\left(1+\ln \frac{t}{r}\right)^{m} \frac{\operatorname{essinf}_{t<\tau<\infty} \varphi_{1}\left(x_{0}, \tau\right) \tau^{\frac{\gamma}{p}}}{\gamma\left(\frac{1}{q_{1}}-\left(\sum_{i=1}^{m} \lambda_{i}+\sum_{i=1}^{m} \frac{1}{p_{i}}\right)\right)+1} d t}^{t} \\
& \leq C\|f\|_{L M_{p, \varphi, P}^{\left\{x_{0}\right\}}}^{\infty} \varphi_{2}\left(x_{0}, r\right) .
\end{aligned}
$$

Then by (2.10) and (3.3), we get

$$
\begin{aligned}
\left\|\left[\vec{b}, T_{\Omega, \alpha}^{P}\right] f\right\|_{L M_{q_{1}, \varphi_{2}, P}^{\left\{x_{0}\right\}}} & \sup _{r>0} \varphi_{2}\left(x_{0}, r\right)^{-1}\left|E\left(x_{0}, r\right)\right|^{-\frac{1}{q_{1}}}\left\|\left[\vec{b}, T_{\Omega, \alpha}^{P}\right] f\right\|_{L_{q_{1}}\left(E\left(x_{0}, r\right)\right)} \\
\leq & C \prod_{i=1}^{m}\|\vec{b}\|_{L C_{p_{i}, \lambda_{i}, P}^{\left\{x_{0}\right\}}} \sup _{r>0} \varphi_{2}\left(x_{0}, r\right)^{-1} \\
& \times \int_{r}^{\infty}\left(1+\ln \frac{t}{r}\right)^{m} \frac{\|f\|_{L_{p}\left(E\left(x_{0}, t\right)\right)}^{m}}{{ }_{t}\left(\frac{1}{q_{1}}-\left(\sum_{i=1}^{m} \lambda_{i}+\sum_{i=1}^{m} \frac{1}{p_{i}}\right)\right)+1} d t
\end{aligned}
$$




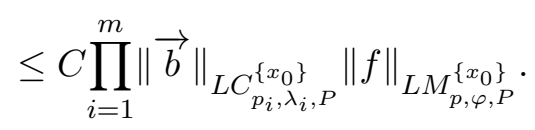

For the case of $q_{1}<s$, we can also use the same method, so we omit the details. Thus, we finish the proof of (1.8).

We are now in a place of proving (1.9) in Theorem 1.5.

Remark 3.3. The conclusion of (1.9) is a direct consequence of the following Lemma 3.4 and (1.8). In order to do this, we need to define an operator by

$$
\left[\vec{b}, \tilde{T}_{|\Omega|, \alpha}^{P}\right](|f|)(x)=\int_{\mathbb{R}^{n}} \prod_{i=1}^{m}\left[\left|b_{i}(x)-b_{i}(y)\right|\right] \frac{|\Omega(x-y)|}{\rho(x-y)^{\gamma-\alpha}}|f(y)| d y \quad 0<\alpha<\gamma,
$$

where $\Omega \in L_{s}\left(S^{n-1}\right), 1<s \leq \infty$, is $A_{t}$-homogeneous of degree zero in $\mathbb{R}^{n}$.

Using the idea of proving Lemma 2 in [4] (see also [9]), we can obtain the following pointwise relation:

Lemma 3.4. Let $0<\alpha<\gamma$ and $\Omega \in L_{s}\left(S^{n-1}\right), 1<s \leq \infty$, be $A_{t}$-homogeneous of degree zero. Then we have

$$
M_{\Omega, \vec{b}, \alpha}^{P} f(x) \leq\left[\vec{b}, \tilde{T}_{|\Omega|, \alpha}^{P}\right](|f|)(x) \quad \text { for } x \in \mathbb{R}^{n} .
$$

In fact, for any $t>0$, we have

$$
\begin{aligned}
{\left[\vec{b}, \tilde{T}_{|\Omega|, \alpha}^{P}\right](|f|)(x) } & \geq \int_{\rho(x-y)<t} \prod_{i=1}^{m}\left[\left|b_{i}(x)-b_{i}(y)\right|\right] \frac{|\Omega(x-y)|}{\rho(x-y)^{\gamma-\alpha}}|f(y)| d y \\
& \geq \frac{1}{t^{\gamma-\alpha}} \int_{E(x, t)} \prod_{i=1}^{m}\left[\left|b_{i}(x)-b_{i}(y)\right|\right]|\Omega(x-y)||f(y)| d y .
\end{aligned}
$$

Taking the supremum for $t>0$ on the inequality above, we get

$$
\left[\vec{b}, \tilde{T}_{|\Omega|, \alpha}^{P}\right](|f|)(x) \geq M_{\Omega, \vec{b}, \alpha}^{P} f(x) \quad \text { for } x \in \mathbb{R}^{n} .
$$

From the process proving (1.8), it is easy to see that the conclusions of (1.8) also hold for $\left[\vec{b}, \tilde{T}_{|\Omega|, \alpha}^{P}\right]$. Combining this with Lemma 3.4, we can immediately obtain (1.9), which completes the proof.

\section{References}

[1] D.R. Adams, Morrey spaces, Lecture Notes in Applied and Numerical Harmonic Analysis, Birkhäuser/Springer, Cham, 2015.

[2] A.P. Calderón and A. Torchinsky, Parabolic maximal functions associated with a distribution, Adv. Math. 16, 1-64, 1975.

[3] R.R. Coifman and G. Weiss, Analyse harmonique non-commutative sur certains espaces homogènes, Étude de certaines intégrales singuliàres, Lecture Notes in Mathematics, 242, Berlin-New York: Springer-Verlag, 1971, (in French).

[4] Y. Ding, Weak type bounds for a class of rough operators with power weights, Proc. Amer. Math. Soc. 125, 2939-2942, 1997.

[5] E. Fabes and N. Riviére, Singular integrals with mixed homogeneity, Stud. Math. 27, 19-38, 1966.

[6] G.B. Folland and E.M. Stein, Hardy Spaces on homogeneous groups, Math. Notes 28, Princeton Univ. Press, Princeton, 1982. 
[7] F. Gürbüz, Parabolic sublinear operators with rough kernel generated by parabolic Calderón-Zygmund operators and parabolic local Campanato space estimates for their commutators on the parabolic generalized local Morrey spaces, Open Math. 14 (1), 300-323, 2016.

[8] F. Gürbüz Parabolic generalized local Morrey space estimates of rough parabolic sublinear operators and commutators, Adv. Math. (China) 46 (5), 765-792, 2017.

[9] F. Gürbüz, Some estimates for generalized commutators of rough fractional maximal and integral operators on generalized weighted Morrey spaces, Canad. Math. Bull. 60 (1), 131-145, 2017.

[10] C.B. Morrey, On the solutions of quasi-linear elliptic partial differential equations, Trans. Amer. Math. Soc. 43, 126-166, 1938.

[11] F. Soria and G. Weiss, A remark on singular integrals and power weights, Indiana Univ. Math. J. 43, 187-204, 1994.

[12] E.M. Stein, Note on singular integrals, Proc. Amer. Soc. Math. 8, 250-254, 1957.

[13] R.L. Wheeden and A. Zygmund, Measure and Integral: An Introduction to Real Analysis, vol. 43 of Pure and Applied Mathematics, Marcel Dekker, New York, NY, USA, 1977.

[14] Q.Y. Xue, Y. Ding and K. Yabuta, Parabolic Littlewood-Paley g-function with rough kernel, Acta Math. Sin. (Engl. Ser.) 24 (12), 2049-2060, 2008. 